\title{
Near-Optimal Disjoint-Path Facility Location Through Set Cover by Pairs *
}

\author{
David S. Johnson ${ }^{\dagger} \quad$ Lee Breslau ${ }^{\ddagger}$ Ilias Diakonikolas ${ }^{\S}$

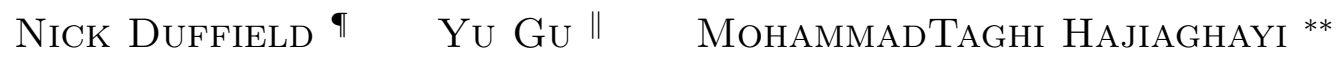 \\ Howard Karloff ${ }^{\dagger \dagger}$ Mauricio G. C. Resende $\ddagger^{\ddagger}$ Subhabrata Sen $\ddagger$
}

July 30,2018

\begin{abstract}
In this paper we consider two special cases of the "cover-by-pairs" optimization problem that arise when we need to place facilities so that each customer is served by two facilities that reach it by disjoint shortest paths. These problems arise in a network traffic monitoring scheme proposed by Breslau et al. and have potential applications to content distribution. The "set-disjoint" variant applies to networks that use the OSPF routing protocol, and the "pathdisjoint" variant applies when MPLS routing is enabled, making better solutions possible at the cost of greater operational expense. Although we can prove that no polynomial-time algorithm can guarantee good solutions for either version, we are able to provide heuristics that do very well in practice on instances with real-world network structure. Fast implementations of the heuristics, made possible by exploiting mathematical observations about the relationship between the network instances and the corresponding instances of the cover-by-pairs problem, allow us to perform an extensive experimental evaluation of the heuristics and what the solutions they produce tell us about the effectiveness of the proposed monitoring scheme. For the set-disjoint variant, we validate our claim of near-optimality via a new lower-bounding integer programming formulation. Although computing this lower bound requires solving the NP-hard Hitting Set problem and can underestimate the optimal value by a linear factor in the worst case, it can be computed quickly by CPLEX, and it equals the optimal solution value for all the instances in our extensive testbed.
\end{abstract}

\section{Introduction}

This paper studies two new facility location problems relevant to questions of Internet traffic monitoring and content distribution. These problems differ from their more standard predecessors in that each customer must be served by two facilities rather than one. In addition, the service routes must be shortest paths and vertex-disjoint.

\footnotetext{
${ }^{*}$ Copyright 2016 AT\&T Intellectual Property. All rights reserved.

${ }^{\dagger}$ Died March 8, 2016. This work was performed when the author was an employee of AT\&T Labs-Research.

${ }^{\ddagger}$ AT\&T Labs-Research, 1 AT\&T Way, Bedminster, NJ 07921.

${ }^{\S}$ School of Informatics, University of Edinburgh, 10 Crichton Street, Edinburgh EH8 9AB, Scotland. This work was performed when the author was a student at Columbia University, and was partially supported by AT\&T.

『Department of Electrical and Computer Engineering, Texas A\&M University, College Station, TX 77843. This work was performed when the author was an employee of AT\&T Labs-Research.

"Amazon Web Services, 1200 12th Avenue South, Seattle, WA 98144. This work was performed when the author was a student at the University of Massachusetts, and was partially supported by AT\&T.

${ }^{* *}$ Computer Science Department, University of Maryland, A.V. Williams Bldg., Room 3249, College Park, MD 20742. This work was performed when the author was an employee of AT\&T Labs-Research.

${ }^{\dagger \dagger}$ This work was performed when the author was an employee of AT\&T Labs-Research.

${ }_{\ddagger}^{\ddagger}$ Amazon.com, 300 Boren Avenue North, Seattle, WA 98109. This work was performed when the author was an employee of AT\&T Labs-Research.
} 
More specifically, suppose we are given a network modeled as an arc-weighted, strongly connected directed graph $G=(V, A)$, together with sets $C \subseteq F \subseteq V$, where $C$ is the set of customer locations and $F$ is the set of potential facility locations. For each pair $(c, f), c \in C$ and $f \in F$, let $P(c, f)$ be the set of all shortest paths from $c$ to $f$ in $G$, which is nonempty by our strong connectivity assumption (and would be so for typical computer networks).

Definition 1. Suppose $c \in C$ is a customer location and $\left\{f_{1}, f_{2}\right\}$ is a pair of potential facility locations in $F-\{c\}$. Then $\left\{f_{1}, f_{2}\right\}$ covers $c$ in a pathwise-disjoint fashion if there exist paths $p_{1} \in P\left(c, f_{1}\right)$ and $p_{2} \in P\left(c, f_{2}\right)$ that have no common vertex except $c$. Such a pair covers $c$ in a setwise-disjoint fashion if no path in $P\left(c, f_{1}\right)$ shares a vertex (other than $c$ ) with any path in $P\left(c, f_{2}\right)$.

Definition 2. A subset $F^{\prime} \subseteq F$ is called a pathwise-disjoint (respectively, setwise-disjoint) cover for $C$ if, for every $c \in C-F^{\prime}$, there is a pair $\left\{f_{1}, f_{2}\right\} \subseteq F^{\prime}-\{c\}$ such that $\left\{f_{1}, f_{2}\right\}$ covers $c$ in a pathwise-disjoint (respectively, setwise-disjoint) fashion. (Note that if $c \in F^{\prime}$, we are assuming that c covers itself, and hence the set $C$ is a valid cover for itself.)

The two problems we study are defined as follows:

Definition 3. In Pathwise-Disjoint Facility Location (PDFL), we are given $G, C, F$, and asked to find a pathwise-disjoint cover of minimum size for $C$. SETwiSE-DiSJOINT FACILITY LOCATION (SDFL) is the same problem except that the cover must be setwise-disjoint.

The Pathwise- and Setwise-Disjoint Facility Location problems arise in a variety of networking contexts. Our primary motivation for studying them comes from a scheme proposed in Gu et al. (2008) for active monitoring of end-to-end network performance, which we shall describe in Section 2. However, both variants have a simple alternative motivation in terms of an idealized content distribution problem, which we shall use to help motivate the definitions. Suppose we wish to distribute data, such as video-on-demand, over a network that connects our service hubs but does not provide a rapid method for repairing link or vertex failures. Suppose further that the service interruptions caused by such failures would be costly to us, and that we want our distribution process to be relatively robust against them. A common standard of robustness is immunity to any single vertex or link failure (as for instance might result from an accidental cable cut). To guarantee such resilience, we would need to place multiple copies of our data source in the network, but because of the costs of hosting such copies, we would like to minimize the number of such hosting sites that we deploy, rather than placing a copy of the data at each service hub.

PAThwise-Disjoint FACILITy Location models this application as follows. The network $G=(V, A)$ is the underlying fiber network linking various service hubs, with the directions of the arcs all reversed, so that $P(c, f)$ is the set of all shortest paths from $f$ to $c$ in the original network, rather than those from $c$ to $f$. The set $C$ of customer locations is the set of service hubs that need access to the data. The set $F$ of facility locations is the set of potential sites where the data can be hosted, which we assume includes the service hubs and possibly other network vertices. If we assume the standard Internet default that shortest paths should be used for routing, the sets $P(c, f)$ now correspond to the paths in the fiber network over which we can route content from facility $f$ to customer $c \neq f$. If we further assume that link capacity is not an issue, then the pathwise-disjoint cover of minimum size for $C$ represents the minimum-cost choice of hosting locations for our data, subject to the constraint that no single vertex or link failure can disconnect a (nonfailed) service hub from all the data sources.

Setwise-Disjoint Facility Location models the variant of this application in which we do not have control over the routing, but instead must rely on the network to do our routing for us. Many Internet Service Providers (ISP's) route packets within their networks using a shortestpath protocol such as OSPF or IS-IS. In such protocols, packets must be routed along shortest paths, where the weight (length) of an arc is set by the network managers so as to balance traffic and optimize other performance metrics. If there is more than one shortest path leaving a given router for a given destination, then the traffic is split evenly between the alternatives. This can 
be of further help in balancing traffic, and so traffic engineers may specifically set weights that yield multiple shortest paths between key routers. The actual splitting is performed based on computing hash functions of entries in a packet's header (such as the source and destination IP addresses). These functions are themselves randomly chosen, are subject to change at short notice, and are typically not available to us. Thus when there are multiple shortest paths, although contemporaneous packets from a given router to the same destination are likely to follow the same path, the actual route chosen may not be readily predictable. All we know is that it must be a member of the set $P(c, f)$ of all shortest paths from $c$ to $f$. This means that the only way to guarantee vertex-disjoint paths to a customer $c$ from two facility locations $f$ and $f^{\prime}$ is to restrict attention to pairs $\left(f, f^{\prime}\right)$ such that the corresponding shortest path sets intersect only in $c$, and consequently our problem becomes a SETWISE-DisJoint FACILITY LOCATION problem.

In this paper, we analyze the complexity of the PDFL and SDFL problems and propose and test algorithms for them. A first observation is that both problems can be viewed as special cases of Set Cover By PAirs (SCP), first described in Hassin and Segev (2005).

Set Cover By PAIRs (SCP): Given a ground set $U$ of elements, a set $S$ of cover objects, and a set $T$ of triples $(u, s, t)$, where $u \in U$ and $s, t \in S$, find a minimum-cardinality covering subset $S^{\prime} \subseteq S$ for $U$, where $S^{\prime}$ covers $U$ if for each $u \in U$, there are $s, t \in S^{\prime}$ such that $(u, s, t) \in T$.

PDFL and SDFL can be formulated as SCP by taking $U=C, S=F$, and

$$
T=\{(c, c, c): c \in C\} \cup\left\{\begin{aligned}
&\left(c, f_{1}, f_{2}\right): c \notin\left\{f_{1}, f_{2}\right\} \text { and }\left\{f_{1}, f_{2}\right\} \text { covers } c \text { in a } \\
& \text { pathwise-disjoint (setwise-disjoint) fashion }
\end{aligned}\right\} .
$$

We prove, subject to a complexity assumption, that no polynomial-time algorithm can approximate SCP to within a factor which is $2^{\log ^{1-\epsilon} n}$ for any $\epsilon>0$. The best previous hardness bound for SCP was just SET Cover-hardness (Hassin and Segev, 2005), which implies that no $o(\log n)$ approximation algorithm can exist unless $\mathrm{P}=\mathrm{NP}$ (Raz and Safra, 1997). We then show that SDFL is just as hard to approximate as SCP, and that PDFL is at least as hard to approximate as SET Cover.

These complexity results (assuming their widely-believed hypotheses) rule out both polynomialtime heuristics that are guaranteed to find good covers and the existence of good lower bounds on the optimal cover size that can be computed in polynomial time. Nevertheless, there still may exist algorithms and lower bounds that are useful "in practice." In this paper we describe and experimentally evaluate our candidates for both.

We test four main heuristics. Each uses as a subroutine a standard randomized greedy heuristic (GREEDY) that actually solves the general SCP problem. The first of our main algorithms, GREEDY(400), is the variant of GREEDY that performs 400 randomized runs and returns the best solution found. The second is a genetic algorithm (GENETIC) that uses GREEDY as a subroutine. The third and fourth, Single Hitting Set (SHS) and Double Hitting Set (DHS), apply only in the Setwise-Disjoint case, exploiting the graph structure in ways that are unavailable to us in the path-disjoint case.

The quality of the solutions that the heuristics produce can, for small instances, be evaluated by applying a commercial optimization package (CPLEX ${ }^{\mathrm{TM}}$ Version 11 in our case) to an integer programming formulation of the derived instances of SCP. This is usually feasible when $|F| \leq 150$, although running times grow dramatically with graph size. For the set-disjoint case we introduce a new lower bound that exploits the graphical nature of our problem to create an instance of the Hitting Set problem (a dual to Set Cover) whose optimal solution value can be no greater than the optimal solution value for our problem. Although this lower bound can underestimate the optimal by a linear factor in the worst case, and its computation requires solving an NP-hard problem, it turns out to be easily computed using CPLEX and to give surprisingly good bounds on all our test instances. In fact, it yielded the optimal solution value for all of them, since for each instance at least one of our heuristics produced a solution whose value matched the bound. 
Moreover, when restricted to our test instances with optimized OSPF weights, the Hitting Set solution itself was a feasible (and hence optimal) solution to our original problem. For the PathDisjoint case, our only lower bound is the comparatively weak one of considering the variant of the problem where we drop the requirement that the paths be shortest paths. The optimum for the resulting problem can be computed in linear time, but is substantially below the optimal PDFL solution, where the latter can be computed. However, for these instances, it at least shows us the penalty imposed by our restriction to shortest paths. For larger instances, we can use our GENETIC algorithm to provide some idea of how much we might be able to improve on GreEDY(400) if time were not an issue.

A final algorithmic challenge was that of constructing the derived SCP instances needed by all our heuristics. This involves exploiting shortest path graphs to determine the (often quite large) sets of relevant triples. Significant algorithmic ingenuity is needed to prevent this computation from being a major bottleneck, and we will describe the mathematical observations and algorithmic techniques that make this possible.

\subsection{Outline}

The remainder of the paper is organized as follows: In Section 2 we describe the network monitoring application that motivated our study. In Section 3 we present our complexity results. In Section 4 we present the lower bounds we use for evaluating our cover-creating heuristics, and our algorithms for computing them. This includes the integer programming formulations for computing the true optima for our problems from their Cover-by-Pairs formulations. In Section 5 we describe the heuristics we have devised, as well as the (nontrivial) algorithms we use to convert our problems to their Cover-by-Pairs formulations. Our test instances are described in Section 6, and our experiments and their results are summarized in Section 7 . We conclude in Section 8 with a discussion of further research directions, including preliminary results for significant variants on our problems, such as the cases where not all members of $C$ need be in $F$, and where facility locations have costs and we wish to find a minimum-cost cover.

\subsection{Related Work}

This paper is, in part, the journal version of a conference paper (Breslau et al., 2011). However, it contains substantial amounts of new material, including the new SDFL lower bound and related algorithms, a faster version of the Genetic algorithm, details of the omitted proofs, key implementation details of our algorithms, and more detailed experimental results and analysis, including an expansion of the experimental testbed to contain significantly larger synthetic and real-world instances. The only previous work on SET COVER BY PAIRs, as far as we know, is that of Hassin and Segev (2005), which is theoretical rather than experimental. That paper considers two applications that were significantly different from those introduced here, and, from a worst-case point of view, much easier to approximate. The paper also introduces a variant of the Greedy algorithm studied here for the general SCP problem and analyzes its (poor) worst-case behavior.

\section{Our Motivating Application: Host Placement for End-to-End Monitoring}

In this section, we describe the monitoring application of $\mathrm{Gu}$ et al. (2008) that motivated this paper, an application that is more realistic than the content distribution application mentioned in the previous section, but also more complicated. We describe it in some detail here to better motivate our study, and also to present a key new lemma that is actually needed to guarantee that the proposed monitoring scheme provides valid results.

Suppose we are an Internet Service Provider (ISP) and provide "virtual private network" (VPN) service to some of our customers. In such a service, we agree to send traffic between various locations 
specified by the customer, promising to provide a certain level of service on the connections, but not specifying the actual route the packets will take. (The actual routing will be done so as to optimize the utilization of our network, subject to the promised levels of service.) Our network is a digraph $G=(V, A)$, in which the vertices correspond to routers and the arcs to the links between routers. A key service quality metric is packet loss rate (the fraction of packets on a path that fail to reach their destination). Let $p\left(r_{1}, r_{2}\right)$ denote the probability that a packet sent from router $r_{1}$ to router $r_{2}$ will successfully arrive. Our goal is to obtain estimates for $p\left(r_{i}, r_{j}\right)$ for a collection of customer paths $P_{r_{i}, r_{j}}$. Note that, in contrast to our content distribution application, we here do not worry about links' failing (which would cause re-routing), but merely about their underperforming.

One way to measure the loss rate on the path in our network from router $r_{1}$ to router $r_{2}$ is to attach extra equipment to the routers, use the equipment at $r_{1}$ to send special measurement packets to $r_{2}$, and use the equipment at $r_{2}$ to count how many of the packets arrive. If $N$ packets are sent and $N^{\prime}$ arrive, then $N^{\prime} / N$ should be a good estimate for $p\left(r_{1}, r_{2}\right)$, assuming $N$ is sufficiently large. Unfortunately, the process of authorizing, installing, and maintaining the extra equipment can be time-consuming and expensive. Thus, this scheme may not be practical in a large network with hundreds or thousands of distinct path endpoints. For this reason, Gu et al. (2008) proposed an alternative scheme that may yield a substantial reduction in the total amount of monitoring equipment needed.

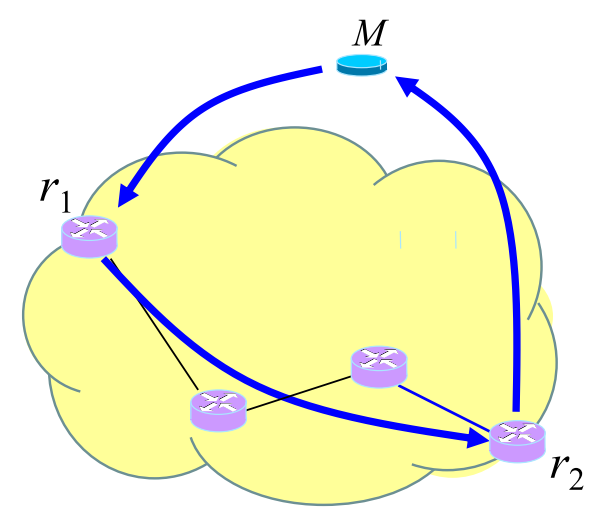

Figure 1: The centralized monitoring scheme of Gu et al. (2008).

In this new scheme, all the monitoring is initiated from a single special measurement vertex $M$, as originally proposed in Burch and Chase (2005) and Breslau et al. (2006). See Figure 1. To measure loss on the path from vertex $r_{1}$ to vertex $r_{2}$, the equipment at $M$ sends a packet on a circular path that first goes from $M$ to $r_{1}$ (the hop-on path), then traverses the path from $r_{1}$ to $r_{2}$, and finally returns from $r_{2}$ to $M$ (the hop-off path). Let us make the following assumptions:

1. Packets are only dropped by arcs, not by vertices. (This is a close approximation to reality in modern-day networks, where an arc models the system consisting of the physical wire/fiber connecting its endpoints, together with the line card at each of its ends.)

2. The three paths $P_{M, r_{1}}, P_{r_{1}, r_{2}}$, and $P_{r_{2}, M}$ are pairwise arc-disjoint. (As we shall show below, this will typically be true under shortest-path routing.)

3. Loss rates on different arcs are independent of each other. (This is somewhat less realistic, but is approximately true except in heavily-loaded networks.)

Then if $N$ packets are sent on the circular path $P_{M, r_{1}, r_{2}, M}$, the expected number $N^{\prime}$ of packets successfully making the roundtrip will be $N^{\prime}=N p\left(M, r_{1}\right) p\left(r_{1}, r_{2}\right) p\left(r_{2}, M\right)$. Thus if we measure $N^{\prime}$ and have good estimates for $p\left(M, r_{1}\right)$ and $p\left(r_{2}, M\right)$, we will have the estimate

$$
p\left(r_{1}, r_{2}\right)=\frac{N^{\prime} / N}{p\left(M, r_{1}\right) p\left(r_{2}, M\right)} .
$$




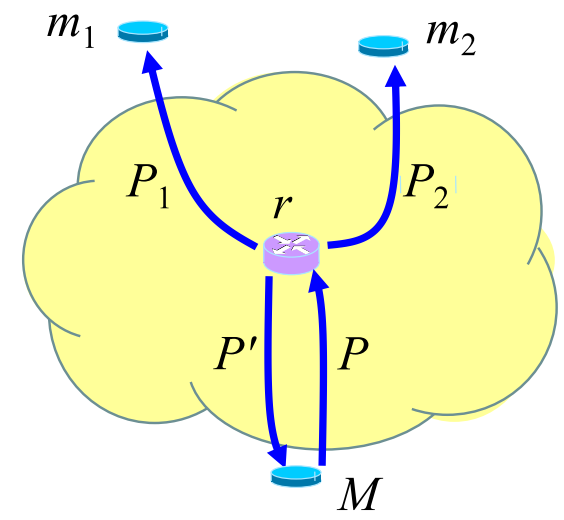

Figure 2: Scheme of Gu et al. (2008) for measuring loss rate of hop-on and hop-off paths.

Thus we have reduced the problem of measuring the loss rates for a collection of paths between arbitrary vertices to that of measuring the loss rates on a collection of round-trip paths and estimating the loss rates for a collection of hop-on and hop-off paths, all of which either begin or end at $M$.

Breslau et al. (2006) proposed that these loss rates for a given path endpoint $r$ be estimated by sending packets along an $(M, r, M)$ circuit and, if, here, $N$ packets were sent and $N^{\prime}$ received, concluding that $p(M, r)=p(r, M)=\sqrt{N^{\prime} / N}$. Unfortunately, this assumes that Internet performance is symmetric, which it definitely is not. A quite accurate way to measure the loss rates would of course be to put equipment at both ends of each of the hop-on and hop-off paths, but this method would require installing equipment at just as many routers as in the original scheme for measuring the $P_{r_{1}, r_{2}}$ paths directly - indeed at one more vertex, since now we need equipment at $M$. Setwise- and Pathwise-Disjoint Facility Location arise in the context of a "tomographic" method proposed by Gu et al. (2008) for estimating loss rates on hop-on and hop-off paths in a potentially much more efficient fashion.

In terms of the facility location problems, the set $C$ of "customer" vertices will consist of the endpoints of the original paths whose loss rates we wish to estimate. The set $F$ of "facility locations" will be these plus those additional vertices that are capable of hosting monitoring equipment. In this context, we will call $F$ the set of (potential) monitoring vertices. Assuming as before that we are in a shortest-path routing regime such as OSPF or IS-IS, the set $P(r, m)$ is the set of all legal routes from $r$ to $m$.

We have already observed that, if we install equipment at $r$ itself, it is straightforward to estimate the loss rates $p(M, r)$ (for the hop-on path to $r$ ) and $p(r, M)$ (for the hop-off path from $r$ ) - simply send packets between $M$ and $r$ and counting the number of them that successfully arrive at their destinations. Suppose $r$ is a path endpoint without equipment and $\left(m_{1}, m_{2}\right)$ is a pair of monitoring vertices that cover $r$ in a pathwise-disjoint fashion. We will now explain how, by installing monitoring equipment at $m_{1}$ and $m_{2}$, we can estimate both loss rates. See Figure 2 . Assuming we are allowed to specify the routing paths from $r$ to $m_{1}$ and $m_{2}$, the fact that $m_{1}$ and $m_{2}$ cover $r$ in a pathwise-disjoint fashion means that we can pick legal routing paths $P_{1}$ and $P_{2}$ from $r$ to $m_{1}$ and $m_{2}$, respectively, that are vertex-disjoint except for $r$ (and hence arc-disjoint). Moreover, as we shall see, we also have that the two paths $P_{1}$ and $P_{2}$ are arc-disjoint from the path $P$ from $M$ to $r$, which itself is arc-disjoint from the path $P^{\prime}$ from $r$ to $M$ (under reasonable assumptions).

This is a consequence of the following lemma, whose additional assumptions have to do with the arc weights used by OSPF and IS-IS in their shortest path computations. These weights are set by traffic engineers to help balance traffic loads and normally obey certain restrictions. First, they are positive integers. Second, in practice networks are typically symmetric directed graphs, in that the 


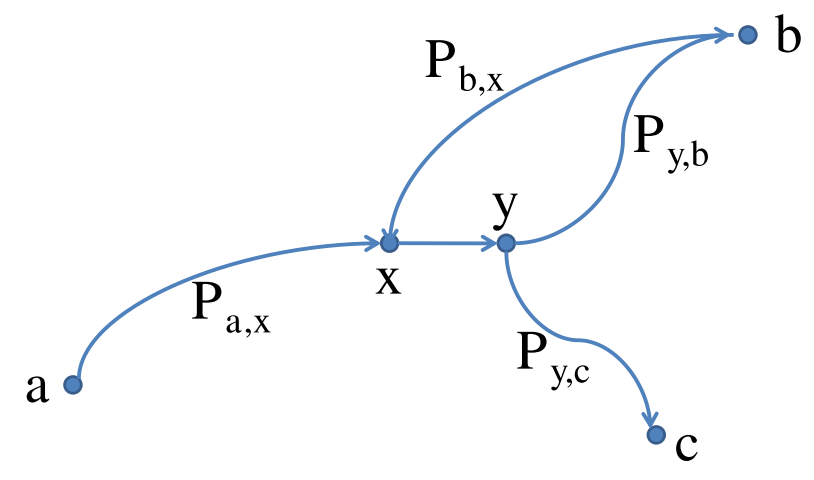

Figure 3: Illustration for proof of Lemma 1.

digraph contains an arc $(a, b)$, then it must also contain arc $(b, a)$, and we assume that the weights $w$ for our digraph are themselves symmetric, in that for every arc $(a, b)$, we have $w(a, b)=w(b, a)$. In real world networks, the weights typically are symmetric, and even networks with asymmetric weights have very few arcs where $w(a, b) \neq w(b, a)$.

Lemma 1. Suppose we are given a symmetric directed graph $G=(V, A)$, a weight function $w$ on the arcs that is symmetric and positive, and three vertices $a, b, c$. If $P_{a, b}$ and $P_{b, c}$ are shortest-weight paths in this digraph from $a$ to $b$ and $b$ to $c$, respectively, then they are arc-disjoint.

Proof. Suppose that, contrary to the Lemma, $P_{a, b}$ and $P_{b, c}$ share a common arc $(x, y)$. Then the path $P_{a, b}$ can be broken up into a path $P_{a, x}$ followed by arc $(x, y)$ followed by a path $P_{y, b}$, where $a$ and $x$ may possibly be the same node, as may $y$ and $b$. Similarly the path $P_{b, c}$ can be broken up into a path $P_{b, x}$ followed by arc $(x, y)$ followed by a path $P_{y, c}$. See Figure 3 .

Note that each of the subpaths of these paths must themselves be shortest paths between their endpoints since the overall path is itself a shortest path. For instance the path $P_{x, b}$ that is the concatenation of $(x, y)$ with $P_{y, b}$ is a shortest path from $x$ to $b$. Let us extend the definition of $w$ to paths $P$ by letting $w(P)$ be the sum of the weights of the $\operatorname{arcs}$ in $P$. Then, for instance, $w\left(P_{x, b}\right)=w(x, y)+w\left(P_{y, b}\right)$. If we let $P_{b, y}$ be the path from $b$ to $y$ obtained by reversing the path $P_{y, b}$, then, since weights are symmetric, we must have $w\left(P_{b, y}\right)=w\left(P_{y, b}\right)=w\left(P_{x, b}\right)-w(x, y)=$ $w\left(P_{b, x}\right)-w(x, y)$.

But now consider the path from $b$ to $c$ obtained by concatenating the two paths $P_{b, y}$ and $P_{y, c}$. It has length $w\left(P_{b, y}\right)+w\left(P_{y, c}\right)=w\left(P_{b, x}\right)-w(x, y)+w\left(P_{y, c}\right)$. On the other hand, by hypothesis, the shortest path from $b$ to $c$ has length $w\left(P_{b, x}\right)+w(x, y)+w\left(P_{y, c}\right)$, and is hence $2 w(x, y)$ longer than the length of this $P_{b, y}$ plus $P_{y, c}$ path. Since by hypothesis $w(x, y)>0$, this is a contradiction.

The basic idea of the technique of Gu et al. (2008) for estimating the loss rate $p(M, r)$ using these paths is to send multicast packets from $M$ to $r$ along path $P$, replicate them at $r$, and then send the copies along paths $P_{1}$ and $P_{2}$ to $m_{1}$ and $m_{2}$, respectively. After this, $m_{1}$ and $m_{2}$ report back to $M$ (using a guaranteed-delivery service such as TCP) as to which packets arrived. Based on this information, $M$ estimates $p(M, r)$. The loss rate $p(r, M)$ can be estimated by sending packets along the $(M, r, M)$ loop and counting the number that arrive back at $M$, using the fact that the 
loss rate for the loop should be $p(M, r) p(r, M)$. (We note that a result like Lemma 1 is needed if this method is to provide reliable estimates, a fact not observed in Gu et al. (2008), which contained no such result.)

This scheme may require two monitoring hosts to measure the hop-on and hop-off rates for a path endpoint $r$, rather than the single one that would be required if we placed the monitoring equipment at vertex $r$ itself. However, the scheme has the potential advantage that a given monitoring vertex can be re-used to handle many different path endpoints. Thus there could be a substantial net overall savings in the total number of monitoring vertices used, and hence in equipment and operational cost.

As stated, the problem of finding a minimum-sized set of monitoring vertices at which to place equipment so that we can estimate loss rates for all hop-on and hop-off paths is simply our original PATHWise-Disjoint FACILITy LocATiOn problem. In practice, however, we will most-likely have to rely on the ISP's routing protocol (OSPF or IS-IS) to deliver our packets, and so, as with our the first application, will face the SETwise-Disjoint FACILITy Location problem.

It should be noted that, in contrast to that first application, the necessity for vertex-disjoint paths from $r$ to $m_{1}$ and $m_{2}$, rather than simply arc-disjoint paths, is less clear, since by the previous lemma we can only guarantee that these paths are arc-disjoint from the path from $M$ to $r$. This is a meaningless distinction in the Setwise-Disjoint case, however, in light of the following lemma.

Lemma 2. Suppose $P(c, f)$ and $P\left(c, f^{\prime}\right)$ are the sets of all shortest paths from vertex $c$ to vertices $f$ and $f^{\prime}$, respectively, in a given digraph $G$. Then no path in $P(c, f)$ shares an arc with any path in $P\left(c, f^{\prime}\right)$ if and only if no path in $P(c, f)$ shares a vertex other than $c$ with any path in $P\left(c, f^{\prime}\right)$.

Proof. The no-shared-vertex case trivially implies the no-shared-arc case, so let us assume that there are no shared arcs and argue that there are also no shared vertices other than $c$. Suppose there were a shared vertex $x \neq c$. Then there is a shortest path $P$ from $c$ to $f$ that is the concatenation of a path $P_{c, x}$ from $c$ to $x$ with a path $P_{x, f}$ from $x$ to $f$, both themselves shortest paths, and a shortest path $P^{\prime}$ from $c$ to $f^{\prime}$ that is the concatenation of a path $P_{c, x}^{\prime}$ from $c$ to $x$ with a path $P_{x, f^{\prime}}^{\prime}$ from $x$ to $f^{\prime}$, both themselves shortest paths. But then the paths $P_{c, x}$ and $P_{c, x}^{\prime}$ must have the same length. Thus the path $P^{\prime \prime}$ that is the concatenation of $P_{c, x}$ with $P_{x, f^{\prime}}^{\prime}$ is a shortest path from $c$ to $f^{\prime}$, and must be contained in $P\left(c, f^{\prime}\right)$. Moreover, since $x \neq c$, the path $P_{c, x}$ contains at least one arc $a$. Thus the path $P^{\prime \prime} \in P\left(c, f^{\prime}\right)$ shares the arc $a$ with the path $P \in P(c, f)$, a contradiction.

A detailed description of the implementation of this scheme and the formulas used for estimating $p(M, r)$ and $p(r, M)$ is presented in Gu et al. (2008).

\section{Complexity}

In this section we investigate the computational complexity of PATHWISE- and SETWISE-DisJoINT FACILITY LOCATION, covering both general hardness results and a polynomial-time solvable special case.

\subsection{Hardness of Approximation}

We first observe that the more general Set Cover BY PAIRs problem is not only NP-hard, but also strongly inapproximable in the worst-case. Let $n=|U|$. Hassin and Segev (2005) observed that SCP is at least as hard to approximate as SET Cover, which cannot be approximated to within a $o(\log n)$ factor unless $\mathrm{P}=\mathrm{NP}($ Raz and Safra, 1997). We can prove much a stronger inapproximability result (albeit with a slightly stronger complexity assumption).

Theorem 3. If $\operatorname{NP} \nsubseteq \operatorname{DTIME}\left(n^{O(\operatorname{polylog}(n))}\right)$, no polynomial-time algorithm can be guaranteed to find a solution to SCP that is within a factor of $2^{\log ^{1-\epsilon} n}$ of optimal for any $\epsilon>0$. 
Proof. The theorem follows via an approximation-preserving transformation from the MinREP problem of Kortsarz, who showed the above inapproximability bound to hold for MinREP (Kortsarz, 2001). The reader may readily confirm that, for the type of approximation bound being proved here, it suffices for the transformation to provide a one-to-one correspondence between solutions for the source and target instances (and their values) and take polynomial time (which also causes the blow-up in the size of the constructed instance to be bounded by a polynomial).

In MinReP, we are given a bipartite graph $G$ with vertex set $V=A \cup B$ and edge set $E$, where $|A|=|B|=k q$ for positive integers $k, q$, together with partitions of the vertex sets on each side of the bipartite graph into $k$ groups of $q$ vertices, $A_{1}$ through $A_{k}$ on the left and $B_{1}$ through $B_{k}$ on the right. We are also given an integer $K$. We ask whether there is a subset $V^{\prime} \subseteq V$ with $\left|V^{\prime}\right| \leq K$ such that for every pair $\left(A_{i}, B_{j}\right), 1 \leq i, j \leq k$, if $G$ contains an edge between a vertex in $A_{i}$ and one in $B_{j}$, then so does the subgraph induced by $V^{\prime}$.

We transform MinREP to SCP by letting the items to be covered be the pairs $\left(A_{i}, B_{j}\right)$ where $G$ contains an edge between a member of $A_{i}$ and a member of $B_{j}$. The set of covering objects is $V$, with the item $\left(A_{i}, B_{j}\right)$ covered by all pairs $\{u, v\} \subseteq V$ in which $u \in A_{i}, v \in B_{j}$, and $(u, v) \in E$. There is then a one-to-one correspondence between SCP cover sets and subsets $V^{\prime}$ meeting the MiNREP requirements, with the two sets having the same size. Hence any approximation guarantee for SCP implies an equally good guarantee for MinREP, and so SCP must be at least as hard to approximate.

Theorem 4. The special cases PDFL and SDFL retain much of the complexity of SCP:

1. SDFL is at least as hard to approximate as SCP.

\section{PDFL is at least as hard to approximate as SET COVER.}

Proof. We shall initially prove the results without the restriction that $C \subseteq F$, and then sketch how the proofs can be modified (by appropriate replication) to enforce the restriction.

For Claim 1, we will show how to transform an arbitrary instance of SCP into an equivalent instance of SDFL. Suppose the given instance of SCP consists of sets $U=\left\{u_{1}, \ldots, u_{p}\right\}$ and $S=$ $\left\{s_{1}, \ldots, s_{q}\right\}$ and relation $T \subseteq U \times S \times S$. By the definition of SCP, we may assume without loss of generality that if $\left(u, s_{i}, s_{j}\right) \in T$, then $i \leq j$. In the corresponding instance of SDFL, there are four types of vertices.

1. For each $u \in U$, a customer vertex $c_{u} \in C$.

2. For each $u \in U$, a connector vertex $x_{u}$.

3. For each $s \in S$, a facility location vertex $f_{s} \in F$.

4. For each pair $\left(s_{i}, s_{j}\right), 1 \leq i<j \leq q$, a pair vertex $v_{s_{i}, s_{j}}$.

There are four types of edges, all having length 1. (The constructed digraph is symmetric, so instead of constructing both $(a, b)$ and $(b, a)$, we just construct one undirected edge $\{a, b\}$.)

1. For each customer vertex $c_{u} \in C$, an edge $\left\{c_{u}, x_{u}\right\}$.

2. For each connector vertex $x_{u}$ and each facility location vertex $f_{s}$, an edge $\left\{x_{u}, f_{s}\right\}$.

3. For each pair $\left(s_{i}, s_{j}\right), i<j$, an edge between $c_{u}$ and $v_{s_{i}, s_{j}}$ unless $\left(u, s_{i}, s_{j}\right) \in T$.

4. For each pair $\left(s_{i}, s_{j}\right), 1 \leq i<j \leq q$, the two edges $\left\{v_{s_{i}, s_{j}}, f_{s_{i}}\right\}$ and $\left\{v_{s_{i}, s_{j}}, f_{s_{j}}\right\}$.

We claim that $F^{\prime} \subseteq F$ is a feasible cover for the constructed SDFL instance if and only if $S^{\prime}=\left\{s: f_{s} \in F^{\prime}\right\}$ is a feasible cover for the original SCP instance. Note that in the constructed digraph, there is a path of length two between each customer vertex $c_{u}$ and each facility location vertex $f_{s_{i}}$, i.e., $\left\langle c_{u}, x_{u}, f_{s_{i}}\right\rangle$, and this is the shortest possible path. Thus $P\left(c_{u}, f_{s_{i}}\right)$ consists precisely 
of this path, together with all paths $\left\langle c_{u}, v_{s_{i}, s_{j}}, f_{s_{i}}\right\rangle$ where $i \leq j$ and $\left(u, s_{i}, s_{j}\right) \notin T$ and all paths $\left\langle c_{u}, v_{s_{j}, s_{i}}, f_{s_{i}}\right\rangle$ where $i>j$ and $\left(u, s_{j}, s_{i}\right) \notin T$. Thus the only vertex that a path in $P\left(c_{u}, f_{s_{i}}\right)$ can possibly share with a path in $P\left(c_{u}, f_{s_{j}}\right), i \leq j$, other than $c_{u}$ itself, is $v_{s_{i}, s_{j}}$, which will be in shortest paths from $c_{u}$ to $f_{s_{i}}$ and to $f_{s_{j}}$ if and only if $\left(u, s_{i}, s_{j}\right) \notin T$. Hence a pair $\left\{s_{i}, s_{j}\right\}$ will cover an element $u$ in the SCP instance if and only if the pair $\left\{f_{s_{i}}, f_{s_{j}}\right\}$ jointly covers the customer vertex $c_{u}$ in the constructed SDFL instance, so the optimal solution values for the two instances coincide.

To get an equivalent instance in which $C \subseteq F$, we replicate the above construction $|S|+1$ times, while, for each $s \in S$, identifying the $|S|+1$ copies of vertex $f_{s}$. We then add the $|S|+1$ copies of each customer vertex $c_{u}$ to $F$ to obtain a new set $F_{+}$of facilities, one that now contains all the customer vertices in our expanded instance. Let $F^{\prime}$ be an optimal solution to our original instance. Note that it remains an optimal solution to the expanded instance. Thus if $F_{+}^{\prime}$ is an optimal solution to this new instance, we must have $\left|F_{+}^{\prime}\right| \leq\left|F^{\prime}\right| \leq|F|=|S|<|S|+1$. Thus at least one of the $|S|+1$ copies of our original instance fails to have any of its customer vertices in $F_{+}^{\prime}$. This implies that $F \cap F_{+}^{\prime}$ must be a feasible solution for that copy, and so $\left|F_{+}^{\prime}\right| \geq\left|F^{\prime}\right|$. But this implies that $\left|F_{+}^{\prime}\right|=\left|F^{\prime}\right|$. So the optimal solution value for our expanded instance still equals that for the original instance of SCP. Moreover, the expanded instance can be constructed in polynomial time and has size within a polynomial factor of the size of the original SCP instance. Hence the inapproximability result for SCP carries over to SDFL.

For Claim 2, we again prove the result without the restriction that $C \subseteq F$. The extension to the case where the restriction holds follows by a replication construction similar to that given in the proof of Claim 1. Our transformation is from SET Cover, which we already know cannot be approximated to within a $o(\log n)$ factor unless $\mathrm{P}=\mathrm{NP}(\mathrm{Raz}$ and Safra, 1997). Given a SET COvER instance, we construct an instance of PDFL whose optimal solution differs from the optimal solution to the SET COVER instance by at most 1. Thus a polynomial-time $o(\log n)$ approximation for our problem would imply one for SET COVER.

An instance of SET COVER consists of a ground set $U=\left\{u_{1}, \ldots, u_{n}\right\}$ and a collection $\mathcal{C}=$ $\left\{C_{1}, \ldots, C_{m}\right\}$ of subsets of $U$. Assume without loss of generality that $\cup_{C \in \mathcal{C}} C=U$. Our constructed graph has four types of vertices:

1. For each $i, 1 \leq i \leq n$, a customer vertex $c_{i} \in C$.

2. For each $i, 1 \leq i \leq n$, a connector vertex $x_{i}$.

3. For each $j, 0 \leq j \leq m$, a potential facility vertex $f_{j} \in F$. Note that $f_{0}$ is an added facility, not corresponding to any member of $\mathcal{C}$.

\section{A universal connector vertex $x_{0}$.}

There are four types of (undirected) edges, all having length 1:

1. For $1 \leq i \leq n$, an edge $\left\{c_{i}, x_{i}\right\}$.

2. For each $i, j, 1 \leq i \leq n$ and $1 \leq j \leq m$, such that $u_{i} \in C_{j}$, an edge $\left\{x_{i}, f_{j}\right\}$.

3. For each $i, 1 \leq i \leq n$, an edge $\left\{c_{i}, x_{0}\right\}$.

4. For each $j, 0 \leq j \leq m$, an edge $\left\{x_{0}, f_{j}\right\}$.

Note that for each pair $i, j, 1 \leq i \leq n$ and $1 \leq j \leq m$, there is a path of length two from $c_{i}$ to $f_{j}$ through $x_{0}$, and this is the shortest possible path. Thus for each such $i, j, P\left(c_{i}, f_{j}\right)$ contains only paths of length two. More precisely, by construction, if $u_{i} \notin C_{j}$ it contains just the path $\left\langle c_{i}, x_{0}, f_{j}\right\rangle$, and otherwise it contains that path plus the path $\left\langle c_{i}, x_{i}, C_{j}\right\rangle$.

Suppose first that $\mathcal{C}^{\prime}$ is a feasible solution to our SET Cover instance. Then it is easy to see that $\left\{f_{0}\right\} \cup\left\{f_{j}: C_{j} \in \mathcal{C}^{\prime}\right\}$ is a feasible solution for our problem. Each $c_{i}$ is covered by $f_{0}$ and an $f_{j}$ such that $u_{i} \in F_{j}$, which must exist because $\mathcal{C}^{\prime}$ is a cover. Thus the optimum for our constructed instance is at most one more than the SET Cover optimum. 
Conversely, suppose that $F^{\prime} \subseteq F$ is a feasible solution for our constructed instance. For each customer vertex $c_{i}$, there must be at least one vertex in $F^{\prime}$ that does not equal $f_{0}$, and so $F^{\prime}-\left\{f_{0}\right\}$ must be a set cover for the original instance. Thus the optimum for the original instance is at most the optimum for our constructed instance. As in the proof of Claim 1, we can now, by replication, construct an instance of PDFL where all customers are facilities, and the optimum value remains unchanged. Consequently, the two optima are within one of each other, which implies that a polynomial-time algorithm with $o(\log n)$ approximation guarantee for PDFL would yield one for Set Cover as well.

\subsection{The Special Case of Trees}

In this section, we consider networks derived from trees, where for a given tree $T=(V, E)$ the corresponding network is the symmetric directed graph $G=(V, A)$, with both $(u, v)$ and $(v, u)$ in $A$ whenever $\{u, v\} \in E$. We first note that, for such instances, the two problems PDFL and SDFL coincide. This follows from the fact that for every ordered pair $v, w$ of vertices, there is exactly one simple path from $u$ to $v$, which is of course the shortest path, and so the shortest path is unique. More interestingly, the problems can be solved in polynomial (in fact, linear) time. Indeed, the optimal solution has a simple combinatorial characterization, and can be constructed by a simple tree traversal.

Theorem 5. The following procedure constructs an optimum cover under PDFL.

1. While $T$ contains a leaf vertex $v$ that is not a customer, delete $v$.

2. Return the set $F^{*}$ of leaves of the residual tree as our cover.

Proof. Note that in the residual tree, all leaves are customers and hence facility locations, so $F^{*} \subseteq F$, as required. Every customer in $F^{*}$ is already covered by itself. If there is any customer $v \notin F^{*}$, it must be an internal vertex of the residual tree, and hence there must be at least two edges incident on $v$ in the residual tree. Each such edge must lie on a path to a leaf of the residual tree, and those paths can contain no common vertex other than $v$ because we are dealing with a tree. But all leaves of the residual tree are in $F^{\prime}$, so this means that $v$ has vertex-disjoint shortest paths to at least two members of $F^{\prime}$ and hence is covered by $F^{\prime}$.

\section{Algorithms}

The complexity results of the previous section rule out (assuming widely-believed hypotheses) both polynomial-time heuristics that are guaranteed to find good covers and the existence of good lower bounds on the optimal solution value that can be computed in polynomial time. Nevertheless, there still may exist algorithms and lower bounds that are useful "in practice." In this section and the next, we describe our candidates for both, beginning with lower bounds, which provide us with a standard of comparison for evaluating the quality of the solutions our heuristics provide.

\subsection{Optimal Solutions Using Mixed Integer Programming.}

The best standard of comparison is, of course, the optimal solution value. It is feasible to compute this for small instances using mixed integer linear programming, by essentially viewing our instances of PDFL and SDFL as SET Cover BY PAIRs instances, modified to account for the fact that a customer, in its role as a facility, can cover itself. This allows us to leverage general purpose commercial software for solving our problems.

Our MIP formulation is simple and straightforward. It has a zero-one variable $x_{f}$ for each facility

$f \in F$, where $x_{f}=1$ if $f$ is chosen for our cover. In addition, we have a real nonnegative variable $y_{f, f^{\prime}}$ for each pair $\left\{f, f^{\prime}\right\}$ of facilities, subject to the constraints that $y_{f, f^{\prime}} \leq x_{f}$ and $y_{f, f^{\prime}} \leq x_{f^{\prime}}$. Note that these together imply that $y_{f, f^{\prime}}$ can be positive only if both $x_{f}$ and $x_{f^{\prime}}$ equal 1 (i.e., are 
in the chosen cover). To guarantee that the chosen set is a cover, we have the following constraints, one for each facility $c \in C$.

$$
x_{c}+\sum_{f, f^{\prime}:\left\{f, f^{\prime}\right\} \text { covers } c} y_{f, f^{\prime}} \geq 1
$$

where "covers" is interpreted as "covers in a path-disjoint fashion" for PDFL and as "covers in a set-disjoint fashion" for SDFL. The goal of our MIP is to minimize $\sum_{f \in F} x_{f}$.

We derive the MIP instances from the corresponding PDFL/SDFL instances using the "triple generation" algorithms described at the end of this section, and then attempt to solve them using the version 11.0 CPLEX ${ }^{\mathrm{TM}}$ MIP solver. This MIP approach proved practical for a wide range of "small" instances, enabling us to find optimal solutions to all but two of the instances in our test set with $|F| \leq 150$. Our detailed experimental results will be summarized in Section 7 .

\subsection{A Hitting Set Lower Bound}

For larger instances of the set-disjoint variant of our problem (SDFL), our standard of comparison is derived from a new lower bound, based on the construction of a special instance of the NP-hard Hitting SeT problem that CPLEX finds it particularly easy to solve. The bound depends on the following simple lemma.

Lemma 6. Suppose $G=(V, A)$ is an arc-weighted directed graph, and $u, v, w$ are distinct vertices in $V$. Let $P_{v}$ be a shortest path from $u$ to $v$, and let $x$ be the first vertex encountered on this path. If there is a shortest path $P_{w}$ from $u$ to $w$ that shares a common vertex other than $u$ with $P_{v}$, then there is a shortest path $P_{w}^{\prime}$ from $u$ to $w$ that contains $x$.

Proof. Let $y$ be the first vertex in $P_{w}$, other than $u$, that is also in $P_{v}$. If $y=x$, we are done, so suppose not. Divide $P_{v}$ into subpaths $P_{v, 1}$ from $u$ to $y$ and $P_{v, 2}$ from $y$ to $v$. Note that $x$ remains the first vertex encountered in $P_{v, 1}$. Similarly divide $P_{w}$ into subpaths $P_{w, 1}$ from $u$ to $y$ and $P_{w, 2}$ from $y$ to $w$. Note that all these paths must be shortest paths from their sources to their destinations. Thus, in particular, $P_{v, 1}$ and $P_{w, 1}$ must have the same length since they are both paths from $u$ to $y$. Hence, the concatenated path $P_{w}^{\prime}=P_{v, 1} P_{w, 2}$ must be a shortest path from $u$ to $w$, and it contains $x$.

For a given customer $c$, let $N(c)$ denote the set of all vertices $x$ such that $(c, x) \in A$ - the neighbors of $c$. For each facility $f \neq c$, let $N(c, f)$ be that subset of nodes of $N(c)$ that are included on shortest paths from $c$ to $f$. As a consequence of Lemma 6, a pair $\left\{f_{1}, f_{2}\right\}$ will cover $c$ in a setwise-disjoint fashion if and only if $N\left(c, f_{1}\right) \cap N\left(c, f_{2}\right)$ is empty. Thus a necessary (although not sufficient) condition for $F^{\prime} \subseteq F$ to contain a pair $\left\{f_{1}, f_{2}\right\}$ that covers $c$ is that $\bigcap_{f \in F^{\prime}} N(c, f)=\emptyset$.

We can use this observation to construct an integer program whose feasible set contains all legal setwise-disjoint covers, and hence whose optimal solution provides a lower bound on the size of the optimal set-disjoint cover. As in the MIP for an optimal solution, we have zero-one variables $x_{f}$ for all $f \in F$, with $x_{f}=1$ meaning that facility $f$ is in our chosen cover, and we wish to minimize $\sum_{f \in F} x_{f}$. Now, however, these are the only variables, and we have a different type of constraint, one for each pair of a customer $c$ and a neighbor $x$ of $c$ :

$$
x_{c}+\sum_{f \neq c \text { and } x \notin N(c, f)} x_{f} \geq 1 .
$$

This IP can be viewed as a "Hitting Set" problem, where a facility "hits" a pair $(c, x)$ if $x \notin N(c, f)$ or if $f=c$. For this reason, we call the optimal solution to the IP the hitting set lower bound (HSLB). It is easy to see that computing this bound is NP-hard. Thus its computation is likely to require exponential time in the worst case, and our SDFL hardness of approximation result, which only applies to polynomial-time algorithms, is not applicable. Nevertheless, the Hitting Set Lower Bound can be quite bad: In the worst case it can underestimate the optimal solution value 


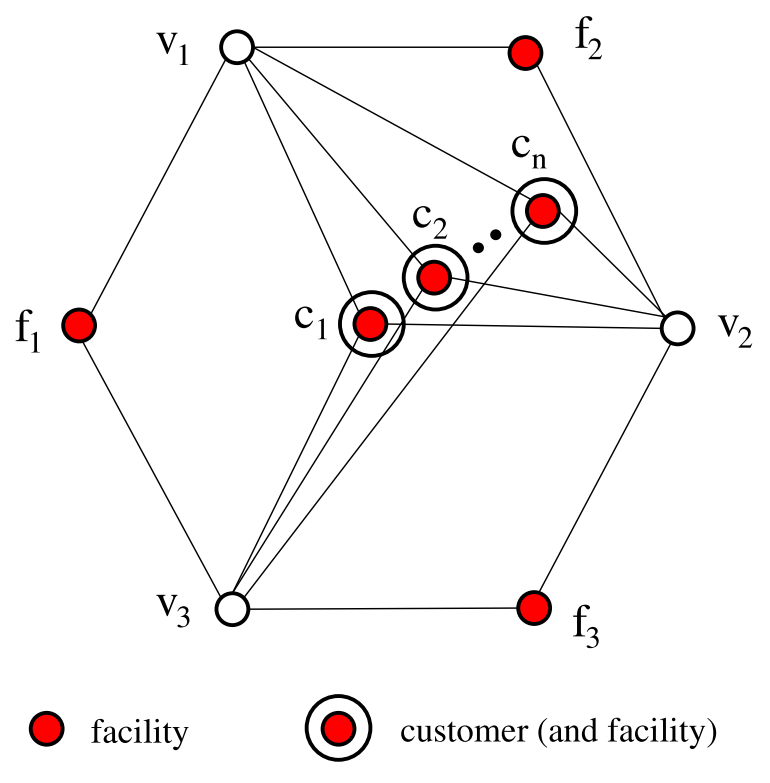

Figure 4: Instances where the HSLB underestimates the optimal cover size by a factor of $n / 3$.

by a linear factor. See Figure 4, which depicts a scheme for instances where the HSLB is too low by a factor of $n / 3$. In this figure there are $n+3$ facility vertices, $f_{1}, f_{2}, f_{3}$, plus the customer vertices $c_{1}$ through $c_{n}$, together with three additional vertices $\left(v_{1}, v_{2}, v_{3}\right)$ that are the neighbors of the customer vertices. Each (undirected) edge in the figure represents a pair of oppositely directed arcs of length 1 . Note that for each neighbor vertex $v$, there is one of the $f_{i}$ whose shortest paths from the customer vertices $c_{i}$ do not contain $v$. Thus, for $n>3$, the solution to the HSLB integer program is the set $\left\{f_{1}, f_{2}, f_{3}\right\}$, for a lower bound of 3 . On the other hand, for each $c_{i}$ and each pair of facilities $\left\{f_{j}, f_{k}\right\} \subseteq\left\{f_{1}, f_{2}, f_{3}\right\}$, we have $N\left(c_{i}, f_{j}\right) \cap N\left(c_{i}, f_{k}\right) \neq \emptyset$ since it contains one of the neighbor vertices. Thus no pair of these vertices covers any customer vertex. In addition, for any customer vertex $c_{h}$ other than $c_{i}$, all three neighbor vertices are on shortest paths from $c_{i}$ to $c_{h}$, so no other customer vertex can help cover $c_{i}$ either. Thus all the customer vertices must cover themselves, and hence the optimal cover has size $n$.

Fortunately, the kind of structure occurring in Figure 4, where $|N(c, f)| /|N(c)|=2 / 3$ for all customer/facility pairs, does not tend to occur in practice. Because of the way networks are designed and the way OSPF weights are typically set, the number of shortest path ties is typically limited, and the ratios $|N(c, f)| /|N(c)|$ are much smaller. In part because of this, the hitting set lower bound is quite good in practice. Indeed, as we shall see in Section 7, the HSLB equaled the optimal solution value for all our SDFL test instances. The solution to the IP was also often a feasible (and hence optimal) solution to the original SDFL instance. Moreover, the hitting set instances themselves turn out to be relatively easy for CPLEX to solve, so we were able to obtain HSLBs for all our SDFL test instances in reasonable time, and usually get optimal solutions as a welcome side-effect.. In light of these observations, we will also include our HSLB code in our comparisons of solution-finding heuristics, albeit one that either produces an optimal solution or no solution at all.

\subsection{An Unconstrained Path-Disjoint Lower Bound}

The previous lower bound applied only to the set-disjoint variant of our problem, and required a linear programming package to compute. For the path-disjoint variant (PDFL), the best polynomialtime computable lower bound we currently have is not nearly as strong in practice, but can be computed in linear time. It applies only to networks that are symmetric directed graphs and hence can be modeled as undirected graphs, but, as remarked above, most real world networks have this property. It equals the minimum cover size for the variant of PDFL where we drop the constraint 


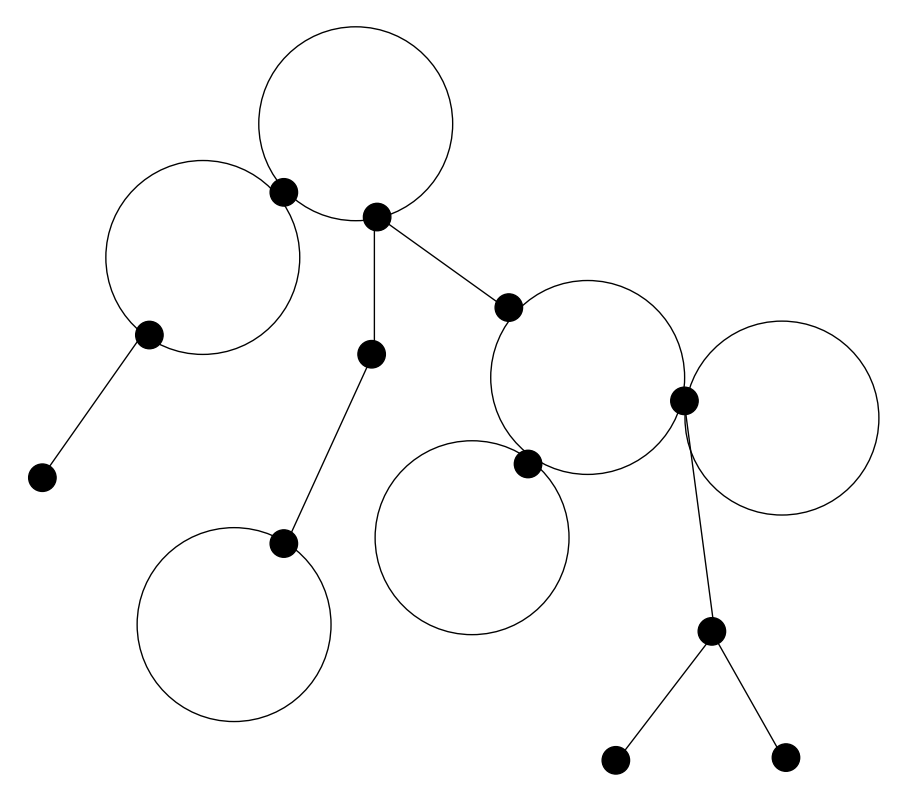

Figure 5: Decomposition into biconnected components.

that our paths be shortest paths, and only insist that they be vertex-disjoint. Let us call the task of finding such a cover as the unconstrained path-disjoint facility location problem (UPDFL). The algorithm for solving the UPDFL problem relies on three observations.

1. Any undirected graph can be decomposed in linear time into its 2-connected components, the resulting structure being treelike, with the 2-connected components playing the role of vertices. (See Figure 5.)

2. If our network is 2-connected, then any pair of facility locations in it will cover all the customers it contains.

3. We already have an easily modifiable algorithm for PDFL on trees (see Section 3.2).

The first observation is attributed to Hopcroft in (Aho et al. 1974), which describes the algorithm in detail. To see the tree structure more clearly, call a vertex an articulation point if deleting it from the graph breaks the graph into two or more separate connected components, and consider the graph $t(G)$ derived from $G$ as follows. The vertices of $t(G)$ are the leaves, the articulation points, and for each 2-connected component $C$, a new vertex $v_{C}$ representing the component. The edges consist of all original edges that did not connect two vertices in the same 2-connected component, together with edges $\left\{u, v_{C}\right\}$ for every pair of an articulation point $v$ and a 2-connected component $C$ that contains it. This graph cannot contain a cycle. If it did, such a cycle would have to contain at least two vertices, each representing either a vertex not in any 2-connected component or a maximal 2-connected set. However, the existence of the cycle would imply that the set consisting of all the vertices and 2-connected components represented in the cycle would itself be 2-connected, a contradiction. Thus $t(G)$ has no cycles, and is a connected graph since by assumption $G$ is. Hence it is a tree.

The proofs of observations (2) and (3) go as follows:

Proof of Observation 2. By definition, if a graph $G$ is 2-connected and $u$ and $v$ are distinct vertices of $G$, then $G$ contains two paths joining $u$ to $v$ whose only common vertices are $u$ and $v$ themselves. Let $u$ and $v$ be two facility locations in our 2-connected graph $G$, and let $c$ be a customer vertex in $G$. If $c \in\{u, v\}$ we are done, so we assume it is distinct from both. Let $P_{1}(u)$ and $P_{2}(u)$ be two vertex-disjoint paths from $u$ to $c$, and similarly, let $P_{1}(v)$ and $P_{2}(v)$ be two vertex-disjoint paths from $v$ to $c$. Such paths exist by the definition of 2-connectivity. We are done 
if either of $P_{1}(v)$ and $P_{2}(v)$ is vertex-disjoint from one of $P_{1}(u)$ and $P_{2}(u)$. So assume that both $P_{1}(v)$ and $P_{2}(v)$ intersect both of $P_{1}(u)$ and $P_{2}(u)$. At most one of them can contain the vertex $u$, so assume without loss of generality that $P_{1}(v)$ does not contain $u$, and let $w$ be the first vertex it contains that is an internal vertex of one of $P_{1}(u)$ and $P_{2}(u)$, say, without loss of generality, $P_{1}(u)$. Then the path that starts with $v$, proceeds along $P_{1}(v)$ until it hits $w$, and then proceeds along $P_{1}(u)$ until it reaches $c$, is vertex-disjoint from $P_{2}(u)$, and once again $u$ and $v$ cover $c$, and this holds for all cases.

Proof of Observation 3. Here is the needed modified version of the algorithm of Section 3.2 . We assume there is at least one customer, as otherwise the empty set is an optimal cover.

Call a 2-connected component a leaf component if it contains only one articulation point. As usual, call a vertex a leaf if it has degree 1 . Note that no leaf can be contained in a 2-connected component, and that all nonleaf vertices that are not in a 2-connected components are articulation points. The internal vertices of a 2 -connected component are all its vertices that are not articulation points. As in the algorithm of Section 3.2, we start with a pruning process. If a vertex is a leaf in the current graph and not a customer, delete it to get a new "current graph." Similarly, if a leaf component has no internal vertices that are customers, delete all its internal vertices, leaving only its (single) articulation point.

After the pruning is complete, the final graph $G^{*}$ is such that every leaf is a customer and every leaf component contains an internal customer. There are two cases to consider:

1. $G^{*}$ contains no leaves or leaf components.

2. $G^{*}$ contains a leaf or leaf component.

The first case implies that $G^{*}$ is a 2-connected graph. By assumption, it must contain at least one customer. If just one, say $v$, then $\{v\}$ is an optimal cover. If more than one, then no single facility can cover all of them, so pick any two customer vertices, say $u$ and $v$, and $\{u, v\}$ will be an optimal cover by our second observation.

For the second case, first note that since all the leaves are now customers, they must be in any optimal cover. Similarly, any cover must contain at least one facility in the interior to each leaf component. To see this, let $v$ be the articulation point for the component, and $c$ an interior point of the component that is a customer. If our purported cover contains no facilities interior to the component, there must be two paths to $c$ from facilities it does contain, but all such paths must now contain $v \neq c$, and hence cannot be vertex-disjoint. Let $F^{*}$ be a set consisting of all the leaves, together with one internal customer from each remaining leaf component. By the above argument, we know that the optimal cover must be at least of size $\left|F^{*}\right|$. We now argue that $F^{*}$ itself is a cover, and hence an optimal one.

Consider any customer vertex in $G^{*}$ that is not in $F^{*}$ and the corresponding vertex $v$ in the underlying tree $t\left(G^{*}\right)$. This is $v$ itself if $v$ is an articulation point or a vertex not contained in any 2-connected component, and otherwise is $v_{C}$ for the 2-connected component for which $v$ is an internal vertex. Note that every leaf of this tree either is a member $F^{*}$ or corresponds to a 2 -connected component containing a member. Thus if $v$ corresponds to a nonleaf of the tree, it must be covered by $F^{*}$, since it has vertex-disjoint paths to at least two leaves. The only other possibility is if $v$ is an internal vertex of a leaf component $C$ of $G^{*}$. But then $F^{*}$ must contain a vertex $f \neq v$ that is one of $C$ 's internal facility locations, since it contains at least one and does not contain $v$. Let $u$ be the single articulation point in $C$. By our second observation, there are vertex-disjoint paths in $C$ from $v$ to to $f$ and $u$. Since $u$ itself must be connected to some leaf outside of $C$, this means that $v$ has vertex-disjoint paths to two members of $F^{*}$. Thus $F^{*}$ is an optimal cover.

As with the Hitting Set Lower Bound for SDFL, the UPDFL lower bound for PDFL is quite bad in the worst case. For such examples we can restrict ourselves to 2-connected graphs, since, as implied by the arguments above, there is no advantage to allowing nonshortest paths except between vertices in the same 2-connected component. So consider the 2-connected graph schematized in Figure 6, where the ellipsis stands for $N-3$ additional connected vertex pairs in the middle row. 


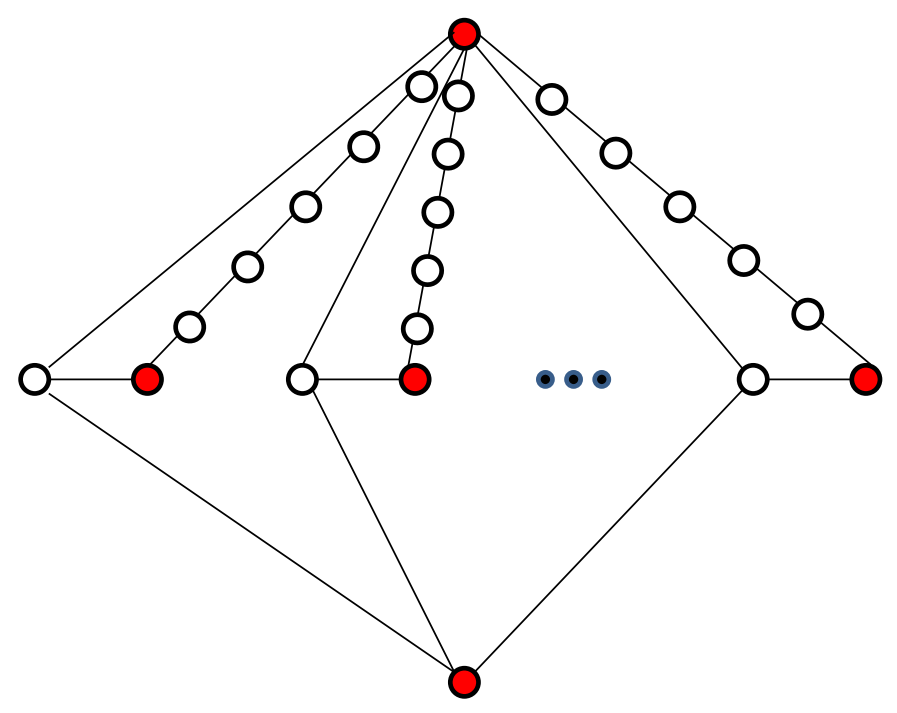

Figure 6: Instances where the UPDFL lower bound underestimates the optimal cover size by a factor that is linear in $n$. The filled-in vertices are the only customers/facilities.

Here the filled-in vertices are the customers and the empty vertices are neither customers nor facilities. There are $N$ customers in the middle row, and every PDFL cover must include all of them, since any shortest path to a target customer in the row from any other customer vertex (in the row above it or below it) has to go though the vertex connected to the target on its left. However, an optimal UPDFL cover consists simply of the two customers at the top and bottom of the figure, since the length-6 path from the top customer to each middle customer shares no vertices with the length-2 path to it from the bottom customer. The total number of vertices is $n=7 N+2$, and so the $N / 2$ ratio between the size of the two covers is linear in $n$.

Note that this is also a lower bound on how large a penalty we can experience by limiting ourselves to shortest paths. For any given instance, if there is a gap between the UPDFL lower bound and the PDFL cover we construct, that gap is the sum of (1) the amount by which the number of monitors used in our constructed solution exceeds the optimal number, plus (2) the penalty (in excess monitors needed) for restricting ourselves to shortest paths. (There may also, of course, be penalties for not using shortest paths, such as added congestion and lessened monitoring accuracy.)

\section{Heuristics}

\subsection{Greedy Heuristic}

The simplest, and most general, heuristic that we tested is a straightforward greedy heuristic, denoted by GREeDY in what follows. This is a heuristic for solving the problem described by the MIP in Section 4.1, and can be applied both to path-disjoint and set-disjoint versions of the problem. It consists of three phases.

1. Construct the triples $\left\{\left(c, f, f^{\prime}\right):\left\{f, f^{\prime}\right\}\right.$ covers $c$ in a path-disjoint (set-disjoint) fashion $\}$ and store them in appropriate data structures.

2. Initialize our cover $F^{\prime} \subseteq F$ to the empty set. Then, as long as $F^{\prime}$ does not cover all $c \in C$, find an $f \in F-F^{\prime}$ such that $F^{\prime} \cup\{f\}$ covers a maximum number of additional elements. Since all customers are facilities, there is guaranteed to be a facility that will cover at least one more customer.

3. Minimalize the cover by testing each facility in the cover in turn to see if its removal would still leave a valid cover, and if so remove it. 
Note that, except for the fact that we allow a customer to cover itself, this is essentially a greedy algorithm for the SET COVER BY PAIRS problem. It differs from the "Greedy" algorithm of Hassin and Segev (2005) for that problem, however. The latter does not include a minimalization phase, and in its growth phase it chooses the best pair to add. It is hence likely to be significantly slower and less effective in practice than our greedy variant.

Our implementation of GREEDY uses randomized tie-breaking. It also allows two options for starting the construction phase: either take a pair that covers the maximum number of customers, or take a random customer, which at least covers itself. We also allow two options for the minimalization phase: either consider facilities in the reverse of the order in which they were added to the cover, or consider them in random order.

None of these options (or combinations of options) seems to dominate any of the others, so here our primary tests cover a multiple-run variant on GREEDY (GREEDY(400)), which performs 400 runs and returns the best solution found. In those 400 runs, we cycle through the four option combinations, using each for 100 runs. Multiple runs help amortize the cost of triple generation. The latter takes time $\Omega(|C| \cdot|F| \cdot|F|)$ and typically yields a set $T$ of triples that is substantially smaller than the $|C| \cdot|F| \cdot|F| / 2$ upper bound, whereas the time for one construction/minimalization cycle is $O\left(|T|+|F|^{2}\right)$. Moreover, as we shall see, the actual overall running times are not large enough to represent an obstacle in the proposed applications, where the time to deploy a solution will greatly exceed the time to compute it. Our experiments will, however, explore the tradeoffs between robustness and number of iterations.

Aside from the triple-generation, which will be described in Section 5.5, the implementation of GREEDY is fairly straightforward, with two exceptions. First, to avoid the multiple cache misses that would occur if we did many random accesses into the list of triples $\left(c, f, f^{\prime}\right)$, we keep three copies of that list, one sorted by the customer $c$, one by the larger-indexed of $f$ and $f^{\prime}$, and one by the smaller, with pointers into the start of the portion for each choice of the sorting key. The list indexed by $c$ is constructed as part of the triple generation process. The other two lists can be computed in linear time by first precomputing how many triples there are for each key and reserving space for them in the corresponding list, and then maintaining appropriate counters while the lists are being constructed. The relevant portions of the lists for each facility $f$ are accessed at most four times, once at the start, once when (and if) $f$ is added to the cover, once in preparation for minimalization, and once when (and if) $f$ is deleted. Another detail: Because we are storing the data three times, we trade a bit of time to save space, by running the triple generation code twice. The first run is used to compute the number of triples, which then allows us to reserve the correct amount of space for each list of triples, rather than simply pre-allocating room for the maximum number $|C| \cdot|F| \cdot(|F|-1)$ of triples.

Our second nonobvious implementation detail consists of the data structures used to handle minimalization, where we need an efficient way of noticing when facilities are required, that is, can no longer be deleted. Let us call a pair of facilities $\left\{f, f^{\prime}\right\}$ valid for a customer $c$ if the pair covers $c$ in the appropriate fashion, and both its members are still in our current cover. A facility $f$ can become required for one of two reasons:

1. $f$ is also a customer and there are no longer any valid pairs for it.

2. There is a customer $c$ such that every valid pair for $c$ contains $f$.

In order to determine when these situations occur, we keep track of two types of counts. First, for each customer $c$, we keep track of the current number mycount $(c)$ of valid pairs for $c$. This makes it easy to check when the first of the above two conditions occurs. Second, for each customer/facility pair $(c, f)$, we keep track of the current number covercount $(c, f)$ of valid pairs for $c$ that contain $f$. This information is then stored in a set of linked lists, one for each customer $c$ and number $n$ of pairs, where the list for $(c, n)$ contains all those facilities $f$ with covercount $(c, f)=n$. To determine whether the second condition occurs, we simply look at the lists for $(c$, mycount $(c))$ for all customers with mycount $(c)$ less than the size of the current cover, since for a cover of size $s$, there can be at most $s-1$ valid pairs containing the same facility. It is not difficult to see that 
the total time for maintaining the data structures is $O(|T|)$, and the total time for spotting all the changes from nonrequired to required is at most $|C|$ times the number of deletions, which is $O(|C| \cdot|F|)$.

\subsection{Genetic Algorithm}

Genetic algorithms are variants on local search that mimic the evolutionary process of survival of the fittest. Our genetic algorithm, called GEnetic in what follows, uses the "random key" evolutionary strategy proposed by Bean (1994), as modified by Gonçalves and Resende (2011) so as to bias the choices of parents and the operation of crossovers. In this approach, the "chromosomes" that do the evolving are not solutions themselves, but "random-key" vectors from which solutions can be derived. (This is in contrast to traditional genetic algorithms, in which the chromosomes are the solutions themselves.)

As with Greedy which it uses as a subroutine, our Genetic algorithm applies to the MIP formulation of the set-disjoint and path-disjoint versions of the problem, and does not directly exploit any graph-theoretical properties of the underlying network. Let the set of facilities $F=$ $\left\{f_{1}, f_{2}, \ldots, f_{k}\right\}$. In Genetic, each chromosome is a $0-1$ vector $\left(\right.$ gene $_{1}, \ldots$, gene $\left._{k}\right)$ of length $k$. We derive a solution (cover) $F^{\prime}$ from such a chromosome as follows: Start with $F^{\prime}=\left\{f_{i}: g_{\text {gene }}=\right.$ $1,1 \leq i \leq k\}$. If $F^{\prime}$ is already a cover, halt. Otherwise, complete $F^{\prime}$ to a cover using our basic GREEDY algorithm (without randomization or minimalization). The "fitness" of the chromosome is then the size of the resulting cover $F^{\prime}$.

The overall genetic algorithm starts by creating a population of $p$ randomly generated chromosomes, in which each gene has an equal probability of being 0 or 1 . The population then evolves in a sequence of generations. In each generation we start by computing the solution for each member of the population, yielding its fitness. The top $15 \%$ of the population by fitness (the "elite" members) automatically pass on to the next generation's population. In addition, to provide diversity, $10 \%$ of the next generation consists of randomly generated chromosomes, like those generated initially. For the remaining 75\%, we repeat the following "biased crossover" construction: Pick a random member $\left(x_{1}, x_{2}, \ldots, x_{k}\right)$ of the top $15 \%$ of the current generation's population and a random member $\left(y_{1}, y_{2}, \ldots, y_{k}\right)$ of the remaining $85 \%$. The "child" $\left(z_{1}, z_{2}, \ldots, z_{k}\right)$ of this pairing is determined as follows: For each $i$, independently, set $z_{i}=x_{i}$ with probability $70 \%$, and otherwise set $z_{i}=y_{i}$.

This scheme insures that the best solution always survives into the next generation, where it may continue as champion or be dethroned by a better solution. Generations are continued until $q$ have gone by without any improvement in the fitness (cardinality) of the best solution, which we then output. Our code includes the option of minimalizing this final solution, but in practice this never has helped.

In our results for Genetic we typically took $p=\min \{300,|F|\}$ and $q=|V|$. For these and the other parameters of the algorithm (top 15\%, etc.) we chose values based on preliminary experimentation and on intuition derived from applications of this approach to other problems. They appear to yield a good tradeoff between running time and quality of solution for our application, but we make no claim as to their optimality.

In our experiments, Genetic did not prove competitive for the set-disjoint case. The much faster heuristics of the next two sections, which exploit the properties of that case, found optimal solutions for all our test instances. In the path-disjoint case, where, for our larger test instances, multiple-iteration GREEDY is the only competition, and where we have no readily-computable lower

bound that is as good as HSLB is for the set-disjoint case, GEnETIC can at least give us some idea of the extent to which GREEDY(400) can be improved upon.

\section{3. $\quad$ The Single Hitting Set Heuristic (SHS)}

This section describes a simple heuristic that applies to the set-disjoint variant of our problem (SDFL), and is based on Lemma 6 and our Hitting Set lower bound for that variant. Instead of optimally solving the NP-hard Hitting Set problem described in Section 4.2, we find a notnecessarily optimal solution using a straightforward greedy hitting set heuristic: Let $X \subseteq F$ denote 
the hitting set we are building, initially the empty set. While $X$ is not a valid hitting set, repeatedly add that facility that hits the most as-yet-unhit (customer,neighbor) pairs, ties broken randomly, where a facility $f$ hits a pair $(c, v)$ if either $f=c$ or $v \notin N(c, f)$. Once $X$ is a valid hitting set, we check whether it is also a valid cover of all the customers and, if not, we extend it to one using GREEDY, this time including randomization and minimalization. (Minimalization is applied even if $X$ is itself already a cover.)

As in GreEdy(400) we use this simple heuristic in a multi-iteration scheme, where we take the best solution found. This allows us to exploit the variance introduced by all the randomization - in addition to all the randomized tie-breaking, we also alternate between reverse delete and randomized delete in the minimalization phases. We shall denote this scheme by $\operatorname{SHS}(k)$, where $k$ is the number of iterations, again typically 400 .

Since $\operatorname{SHS}(k)$ is a polynomial-time algorithm, Theorem 4 implies that we cannot prove a polylogarithmic worst-case performance guarantee for it unless a widely-believed conjecture is false. However, in practice the initial hitting set $X$ is typically close to a cover, and so the following provides an informative bound.

Theorem 7. For a given instance $I$ of $S D F L$ based on a graph $G=(V, A)$, let $\mathrm{OPT}(I)$ be an optimal cover, and suppose we run SHS one time, with $X(I)$ being the hitting set generated and $\mathrm{SHS}(I)$ being the final cover. Then, if we set $\Delta=|\mathrm{SHS}(I)|-|X(I)|$, the number of facilities that needed to be added (removed) to turn $X$ into a minimalized cover, we have

$$
\operatorname{SHS}(I) \leq(\ln (|A|)+1) \cdot \mathrm{OPT}(I)+\Delta .
$$

Proof. This follows from the fact that the optimal solution to the hitting set problem of Section 4.2 is a lower bound on OPT $(I)$, together with the result of Johnson (1974) and Lovász (1975) that the greedy algorithm for SET Cover (and hence for HitTing SET) has worst-case ratio at most $\ln n+1$, where $n$ is the size of the set being covered/hit.

Note that the $\ln n+1$ bound for the greedy HitTing SET heuristic is essentially the best possible guarantee for a polynomial-time algorithm for this problem, as follows from Bellare et al. (1993), Feige (1998), and Alon et al. (2006). In practice, however, the greedy HitTing SET heuristic typically returns a set of size much closer than this to the optimal, often only a singledigit percentage above optimal. As we shall see, the performance of $\mathrm{SHS}(k)$ is similarly good.

\subsection{The Double Hitting Set Heuristic (DHS)}

This section describes a second heuristic for SDFL that exploits Lemma 6, as well as the way OSPF weights tend to be set. It involves computing two hitting sets rather than one, but is a good match for SHS, in that both heuristics perform very well and neither consistently dominates the other.

As remarked above, while OSPF can split traffic in the case of multiple shortest $c \rightarrow f$ paths,

the amount of splitting that occurs in practice seems to be limited. The following two definitions provide a new way to quantify this effect.

Definition 4. A potential facility location $f$ is good for customer vertex $c$ if $f \neq c$ and $|N(c, f)|=1$, that is, all shortest $c \rightarrow f$ paths leave $c$ via the same arc.

If there is just one shortest $c \rightarrow f$ path, clearly $f$ is good for $c$, but $f$ may be good for $c$ even when there are many $c \rightarrow f$ paths. Note that if $f$ is good for $c$, then, under OSPF, there can be no splitting at $c$ of the traffic from $c$ to $f$, although splitting at later vertices in the path would be possible.

Definition 5. Let $t$ be an integer, $1 \leq t \leq|F|$. A customer vertex $c$ is $t$-good if there are $t$ or more good potential facility locations $f$ for $c$ (and $t$-bad otherwise). 
For our test instances, inspired or derived from real-world networks and models, a large majority of the customers tend to be $t$-good, even for $t \sim|F| / 2$, and almost all are 1-good. Our Double Hitting Set heuristic (DHS) takes $t$ as a parameter, and is designed to cover the $t$-good customers nearly optimally, via a union $X \cup Y$ of two hitting sets, leaving the $t$-bad customers, if any, to be covered via the GREEDY algorithm. Let $C_{t}$ denote the set of all $t$-good customers. For a given $t$, we shall, when it makes a difference, denote the algorithm by $\mathrm{DHS}_{t}$.

The first step of $\mathrm{DHS}_{t}$ is to choose one good potential facility location $f \in F$ for each $c \in C_{t}$, without choosing too many vertices in total. For each such $c$, let $S_{c}$ be the set consisting of $c$ plus all the good potential facility locations for $c$. By the definition of $t$-good and the fact that $C \subseteq F$, we must have $\left|S_{c}\right| \geq t+1 \geq 1$. We want to choose a small set $X \subseteq F$ such that $X \cap S_{c} \neq \emptyset$ for all $t$-good customers $c$. In other words, we want an $X$ that hits (intersects) all such sets $S_{c}$. We again settle for using the standard greedy HitTing SET heuristic, as described in the previous section, to construct a hitting set $X$ that we hope is near-optimal.

Next, let $C^{\prime} \subseteq C_{t}$ be the set of $t$-good customers $c$ covered by $X$, either because $c \in X$ or because there exist distinct $f_{1}, f_{2} \in X$ such that $c$ is covered by $\left\{f_{1}, f_{2}\right\}$ in a setwise disjoint fashion. Let $C^{\prime \prime}=C_{t}-C^{\prime}$, and for each customer $c \in C^{\prime \prime}$, choose a facility $f_{c} \in X \cap S_{c}$. Note that by definition of $C^{\prime \prime}$, we have $f_{c} \neq c$, so let $x_{c} \in N(c)$ be the unique neighbor of $c$ which is on all shortest paths from $c$ to $f_{c}$. This choice is unique since the fact that $f_{c} \in S_{c}$ implies that $f_{c}$ is good for $c$, and so by definition all shortest paths from $c$ to $f$ leave $c$ through the same arc.

Our second HitTing SET problem is designed so any solution $Y$ will, for each $c \in C^{\prime \prime}$, contain either $c$ itself or a facility $f \neq c$ such that $\left\{f_{c}, f\right\}$ covers $c$ is a setwise disjoint fashion. The HitTING SET problem is specified as follows. For each such customer vertex $c$, let $F_{c}$ consist of $c$ together with all potential facility locations $f \neq c$ for which all shortest $c \rightarrow f$ paths avoid $x_{c}$. Note that by Lemma 6, for any $f \in F_{c}$ (other than $c$ itself), the fact that $f_{c}$ is good for $c$ and the definition of $x_{c}$ together imply that the pair $\left\{f_{c}, f\right\}$ will cover $c$ in the desired fashion. We thus have the following:

Lemma 8. Given $X$ as above, any hitting set $Y$ of $\left\{F_{c}: c \in C^{\prime \prime}\right\}$ is such that $X \cup Y$ is a cover of $C^{\prime} \cup C^{\prime \prime}=C_{t}$, the set of all the t-good customer nodes $c$.

For the DHS algorithm, we also use the greedy Hitting Set heuristic to produce $Y$. At this point, we have a cover $X \cup Y$ for $C_{t}$. DHS concludes by using GREEDY to extend $X \cup Y$ to a minimalized cover $F^{\prime}$ of all the customers, not just the $t$-good ones.

As with SHS, we will consider multi-iteration schemes, $\operatorname{DHS}_{t}(k)$, where we perform $k$ independent runs of $\mathrm{DHS}_{t}$, in each using randomized tie breaking when running the greedy HiTTING SET heuristic and the final GREEDY completion phase, and alternating between reverse and random deletes in the minimalization phases. Our main experiments will be with $\mathrm{DHS}_{1}(400)$ and $\operatorname{DHS}_{\lfloor|F| / 2\rfloor}(400)$, which seemed somewhat complementary in our initial, single-iteration trials. For simplicity, we shall denote the latter by $\operatorname{DHS}_{H}(400)$ (with the $H$ standing for "half").

As with $\operatorname{SHS}(k)$, since $\operatorname{DHS}_{t}(k)$ is a polynomial-time algorithm, Theorem 4 implies that we cannot prove a polylogarithmic worst-case performance guarantee for it unless a widely-believed conjecture is false. However, in practice the initial hitting set $X$ is typically quite small, and often there are very few $t$-bad customers, so meaningful bounds are possible.

Theorem 9. Suppose $1 \leq t \leq|F|$, and let $I$ be an instance of SDFL with at least one $t$-good customer. Let $\mathrm{OPT}_{t}(I)$ be the size of an optimal cover for the set $C_{t}$ of all t-good customers in I. Suppose we run $\mathrm{DHS}_{t}$ one time on $I$, with $X(I)$ and $Y(I)$ being the two hitting sets generated, which, as observed constitute a cover of $C_{t}$. Then,

$$
|X \cup Y| \leq\left(\ln \left(\left|C_{t}\right|\right)+1\right) \cdot \operatorname{OPT}_{t}(I)+|X|
$$

and, if $t \geq|F| / 3$, we have

$$
|X \cup Y| \leq\left(3.47 \cdot \ln \left(\left|C_{t}\right|\right)+2\right) \cdot \mathrm{OPT}_{t}(I)
$$

Note that, for our implementation choice of $t=\lfloor|F| / 2\rfloor$, we have $t \geq|F| / 3$ as long as $|F| \geq 2$. Also, the cover of all of $C$ produced by $\mathrm{DHS}_{t}$ can exceed $|X \cup Y|$ by at most the number $\left|C-C_{t}\right|$ 
of $t$-bad customers, so if almost all customers are $t$-good, the above bounds will reflect the overall performance of DHS. Finally, note that the logarithm here is of $|C|$ or something less, whereas for the analogous Theorem 7 for SHS, the logarithm was over the potentially much larger $|A|$, which might affect the relative constant factors in the bounds.

Proof. Observe that the sets $F_{c}$ in our second hitting set constitute a subset of those used in our Hitting SeT lower bound, restricted to the $t$-good customers. Thus the optimal hitting set for this second problem cannot be larger than $\mathrm{OPT}_{t}(I)$. Thus, by the results of Johnson (1974) and Lovász (1975) and the fact that, by assumption, $C_{t} \neq \emptyset$, we have $|Y| \leq(1+\ln |C|) \cdot \mathrm{OPT}_{t}(I)$, and the first claim follows.

We cannot apply the same argument to the set $X$, since in constructing $X$ we restricted attention to good potential facility locations, and $\mathrm{OPT}_{t}(I)$ need not be so restricted. However, a different argument applies when $t \geq|F| / 3$, which will lead to the proof of our second claim. This choice of $t$ means that, for each $t$-good vertex $c,\left|S_{c}\right| \geq|F| / 3$. Therefore, $\sum_{c}$ is $t$-good $\left|S_{c}\right| \geq\left|C_{t}\right| \cdot|F| / 3$, and so some potential facility location $f$ must be in at least $\left|C_{t}\right| / 3$ of the sets. Consequently, the greedy choice must hit at least that many sets. By essentially the same argument, it must hit at least $1 / 3$ of the remaining unhit sets each time it makes a choice, and so must have completed constructing its hitting set $X$ after $y$ steps, where $(2 / 3)^{y}|C|<1$. This means it suffices that $y>\ln (|C|) / \ln (3 / 2)>2.47 \cdot \ln (|C|)$, and so $|X| \leq 1+2.47 \cdot \ln (|C|)$. The second claim follows.

\subsection{Triple Generation.}

All our cover-generation algorithms require the generation of the set of relevant triples $\left(c, f_{1}, f_{2}\right)$ (set-disjoint or path-disjoint) used by our basic GREEDY algorithm - the algorithms other than GREEDY, either use it as a core-subroutine (GENETIC) or as a fallback device to complete the cover they generate (SHS and DHS). Because this set is potentially of size $\Theta\left(|C| \cdot|F|^{2}\right.$ ), and would naively take significantly more time than that to generate, its computation typically constitutes a significant portion of the running time. In this section we show how the time can be reduced to $O\left(|V|^{3}\right)$ for real-world networks, in both the set- and path-disjoint cases.

In what follows, let $n=|V|$ and $m=|A|$. For real-world data networks, we may assume that $m \leq$ an for some relatively small constant $a$. We also have that $|C| \leq|F| \leq n$, although both $|C|$ and $|F|$ may be proportional to $n$. Let $T_{S}$ and $T_{P}$ be the sets of triples needed by GreEDY in the set-disjoint and path-disjoint cases, respectively.

Let us first consider the set-disjoint case. A triple $\left(c, f_{1}, f_{2}\right)$ is in $T_{S}$ if $c \in C, f_{1}, f_{2} \in F$, and no shortest path from $c$ to $f_{1}$ shares any vertex other than $c$ with any shortest path from $c$ to $f_{2}$. The naive way to test this would be to construct, for each $f_{i}$, the set $S_{i}$ of vertices on shortest paths from $c$ to $f_{i}$, and then testing whether $S_{i} \cap S_{j}=\{c\}$. These sets could conceivably be of size proportional to $n$, yielding a running time that could be proportional to $|C||F|^{2} n$, so the best we can say about the running time of such an algorithm is that it is $O\left(n^{4}\right)$.

However, recall from Lemma 6 that if $S_{i} \cap S_{j}$ contains some vertex other than $c$, then it contains a vertex that is a neighbor of $c$. Thus we may restrict attention to the sets $N_{i}$ of vertices adjacent to $c$ that are on shortest paths to $f_{i}$. To compute these sets we first construct a shortest path graph from $c$ to all other vertices in time $O(m \log n)$, and then for each facility $f$ we can compute $N_{i}$ by a single backward traversal of the shortest path graph from $f$ in time $O(m)$. For each pair $f_{i}, f_{j}$, the intersection test can then be performed in time $O$ (outdegree $(c))$. Amortized over all customers in $C$, the tests for a given pair $f_{i}, f_{j}$ take time $O\left(\sum_{c \in C}\right.$ outdegree $\left.(c)\right)=O(m)$. Thus we get an overall time bound of $O\left(m \log n|C|+m|F| \cdot|C|+m|F|^{2}\right)=O\left(n^{2} m\right)$ under our assumptions that $m$ is $O(n)$.

For the pathwise-disjoint problem, the naive algorithm is even worse, since for a given triple $c, f_{1}, f_{2}$, there may be exponentially many paths of potential length $\Omega(n)$ to compare. Here we must be more clever. We first observe that we can actually reduce the test to a simple network flow problem, by augmenting the shortest path graph with a new vertex $t$, adding arcs from $f_{1}$ and $f_{2}$ to $t$, giving all vertices and edges capacity one, and asking whether there is a flow of value 2 from 
$c$ to $t$. Next, we observe that this is a particularly easy flow problem, which can be solved in linear time as follows.

As before, for a given customer $c$ we begin by computing a shortest path graph from $c$ to all other vertices in time $O(m \log n)$, to be amortized over all facilities. Set the capacities of all the arcs to 1 . We then add a new vertex $t$, and edges of length and capacity 1 from all facilities $f$ to $t$. To handle a given candidate pair $\left(f_{1}, f_{2}\right)$, we pick some shortest path from $c$ to $f_{1}$ using the shortest path graph, and extend it to a shortest path from $c$ to $t$ by adding the link from $f_{1}$ to $t$. This yields a flow of 1 from $c$ to $t$. Now add a link of capacity 1 from $f_{2}$ to $t$. There will be disjoint paths from $c$ to $f_{1}$ and to $f_{2}$ if and only if there is now a flow of value 2 from $c$ to $t$, and such a flow will exist if and only if there is an augmenting path from $c$ to $t$ in the graph. This can be found in linear time by a breadth-first search in the residual graph, where each arc $(u, v)$ in the first path is replaced by an $\operatorname{arc}(v, u)$ of capacity 1 .

Finally, we observe that, having fixed the shortest path to $t$ through $f_{1}$ and constructed the residual graph, we can actually solve the network flow problems for all pairs $\left(f_{1}, f_{i}\right)$ in parallel with a single breadth-first search. The overall running time thus becomes $O(m \log n|C|+m|C| \cdot|F|)=$ $O\left(n^{2} m\right)$, once again $O\left(n^{3}\right)$, assuming $m=O(n)$.

What we have just described is actually the algorithm for generating path-disjoint triples when "disjoint" only means "arc-disjoint." To get vertex-disjointness as well, one replaces each vertex $v$ in the shortest path graph be a directed $\operatorname{arc}\left(v_{\text {in }}, v_{\text {out }}\right)$, with each $\operatorname{arc}(u, v)$ replaced by $\left(u, v_{\text {in }}\right)$ and each $\operatorname{arc}(v, u)$ replaced by $\left(v_{\text {out }}, u\right)$.

In conclusion, we note that for our HSLB code in the set-disjoint case, some of the above work can be avoided, as the triples need only be considered implicitly. To construct the HitTing SET linear program, we need only consider (customer,neighbor,facility) triples, at an amortized cost (and space) of $O(m n)$. The same triples can be reused in the verification phase, where we check to see whether the computed hitting set is actually a feasible solution to our problem, although now $O\left(m n^{2}\right)$ time may be required.

\section{Network Test Instances}

We tested our algorithms on both synthetic and real-world instances. The two classes modeled different types of data networks and had distinctly different characteristics, enabling us to test the generality of our methods. In this section we will discuss each type of instance, and compare the structural properties of the resulting testbeds.

\subsection{Synthetic LAN/WAN Instances}

Our 630 synthetic instances were designed to reflect the structure of large real-world local- and wide-area networks (LAN's and WAN's) and were sized so that we could study the scalability of our algorithms and the solutions they produce. They were generated via the following four-step process.

1. A transit-stub skeleton graph is generated using the Georgia Tech Internetwork Topology Models (GT-ITM) package (Calvert et al., 1997). We generated 10 graphs each for parameter settings that yielded $|V|=50,100,190,220,250,300,558,984$. (The value of $|V|$ is an indirect result of one's choice of the allowed input parameters.)

2. Traffic demand is generated between all pairs of vertices in the skeleton graph using a gravitational model (described below) with the shortest path metric.

3. Using the heuristic network design algorithms of Buriol et al. (2007), we determine link capacities and OSPF link weights such that all traffic can be routed in a balanced way on the resulting network using the OSPF routing protocol. Links with no traffic routed on them are deleted. 


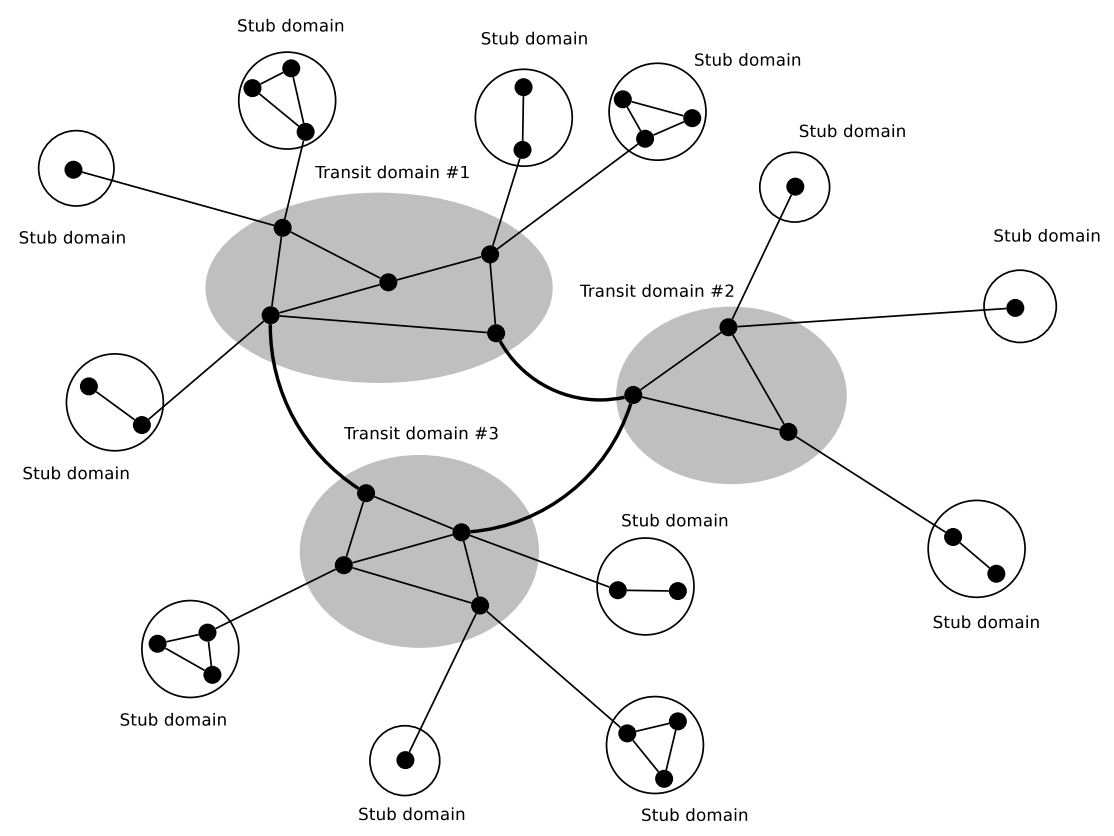

Figure 7: A transit stub network with three transit domains and 16 stub domains.

4. For specified numbers of customer and facility location vertices, the sets $C \subseteq F$ are then randomly generated.

\subsubsection{Step 1: Transit-Stub Skeleton Graphs}

Transit-stub graphs (Calvert et al., 1997) are hierarchical graphs made up of transit vertex components and stub vertex components. The stub node components can be thought of as access networks while the transit node components make up the backbone of the network. See Figure 7.

The GT-ITM package provides a parameterized method for generating such graphs randomly. The relevant papers and documentation are not totally consistent or clear, and the following description is our best guess as to how the networks are generated, based on the documentation and our inspection of selected outputs. The generation process involves many parameters, but we based our generation on the settings used in the illustrative example identified as "file t100" in the documentation file itm-doc.txt available at http://www.cc.gatech.edu/projects/gtitm/, varying only the parameters $T, N_{T}, S, N_{S}$, which determine the number of vertices.

The constructed graphs consist of $T \cdot N_{T}$ transit vertices assigned randomly to $T$ transit domains. It is required that $T \geq 1$ and $N_{T} \geq 1$, and it appears that the generation process starts by placing one transit vertex in each transit domain so that none are empty. In addition, there are $S \cdot T \cdot N_{T}$ stub domains (one per transit vertex), and $S \cdot N_{S} \cdot T \cdot N_{T}$ stub vertices, assigned randomly to the stub domains. Again we must have $S \geq 1$ and $N_{S} \geq 1$ and it appears that the generation process starts by placing one stub vertex in each stub domain. Edges appear to be added to the network as follows:

- Each transit domain has an average of two edges to other transit domains. The endpoints of each edge are chosen randomly from the transit vertices in the two relevant domains. If the chosen edges fail to connect all the transit domains, they are deleted and the experiment is repeated until we do get a connected graph.

- Each transit vertex is associated with an average of $S$ stub domains, and is connected by an edge to a random member of each.

- For each pair of vertices within a given transit domain, there is an edge with probability 0.6. If the resulting choices do not make the transit domain connected, the experiment is repeated until a connected graph results. 


\begin{tabular}{rrrr|rrc}
$T$ & $N_{T}$ & $S$ & $N_{S}$ & $|V|$ & Average $|A|$ & Average Degree \\
\hline 1 & 2 & 3 & 8 & 50 & 91.1 & 3.6 \\
1 & 4 & 3 & 8 & 100 & 177.2 & 3.5 \\
2 & 5 & 3 & 6 & 190 & 284.6 & 3.0 \\
2 & 5 & 3 & 7 & 220 & 360.2 & 3.3 \\
2 & 5 & 3 & 8 & 250 & 435.0 & 3.5 \\
2 & 6 & 3 & 8 & 300 & 531.6 & 3.5 \\
3 & 6 & 3 & 8 & 558 & 1172.2 & 4.2 \\
4 & 6 & 4 & 10 & 984 & 2083.3 & 4.2 \\
\hline
\end{tabular}

Table 1: Input parameters and measured statistics of generated transit-stub graphs.

- For each pair of vertices within a given stub domain, there is an edge with probability 0.42 . If the resulting choices do not make the stub domain connected, the experiment is repeated until a connected graph results.

In addition, it appears that the code also adds a few extra edges between stub domains or between stub and transit domains, even though there should be none, given the parameter settings we chose. We deduce this from the fact that, although the above description suggests that the largest 2-connected component should be of size $\max \left(N_{S}, T \cdot N_{T}\right)$, we typically find that the largest is two to four times larger than that.

Note that this process yields a network with a fixed number $T \cdot N_{T} \cdot S \cdot N_{S}$ vertices, but a random number of edges (some of which may be deleted in Step 3). Before proceeding to Step 2, we convert our graphs to symmetric directed graphs by replacing each edge $\{u, v\}$ with the two arcs $(u, v)$ and $(v, u)$. Since the original graph was designed to be connected, this directed graph will be strongly connected. The GT-ITM generator also produces a length $l(u, v)$ for each edge $\{u, v\}$, which we use in Step 3.

We generated 10 graphs each for eight different sets of parameters, chosen to yield increasing values for $|V|$, from 50 to 984 . In Table 1 we present the parameter choices, the corresponding values for $|V|$, the final average number of edges and the average degree (when the graph is viewed as undirected). The graphs for instances with up to 558 vertices were generated by the 32-bit version of the GT-ITM code, which is apparently no longer supported. Those for the graphs with 984 vertices, which we generated more recently, were produced using the new, 64-bit code from Version 2.33 on ns-allinone, downloaded from http://sourceforge.net/projects/nsnam/files/. This version apparently has a bug, in that the parameters for the edge densities seem to be ignored. Consequently, both the stub and transit domains start out as complete graphs, and their sparsification, while still substantial, is due only to the Step 3 deletions, described in more detail below.

\subsubsection{Step 2: Traffic Demands}

The traffic demands are created via a randomized "gravitational" method from Fortz and Thorup (2000). We first generate random numbers $o(v)$ and $d(v)$ for each vertex $v$, chosen independently and uniformly from the interval $(0,1)$. Here "o" stands for origin and "d" for destination. Then, for each pair of vertices $(u, v)$, we compute $D(u, v)$, the shortest length (in edges) of a path from $u$ to $v$. Let $D \max$ be the largest of these distances. Then, for any ordered pair $(u, v)$ of distinct vertices, we choose a random number $r(u, v) \in(0,1)$ and set the traffic demand from $u$ to $v$ to be the (nonzero) value

$$
t(u, v)=r(u, v) \cdot o(u) \cdot d(v) \cdot e^{-\left(\frac{D(u, v)}{D m a x}\right)} .
$$

Note that, since our network is strongly connected, all of this traffic can be routed. 


\subsubsection{Step 3: OSPF Weights}

We next choose OSPF weights and arc capacities that allow for an efficient routing of the traffic specified by the matrix $t(u, v): u, v \in V$. To do this, we apply the survivable IP network design tool developed by Buriol et al. and described in Buriol et al. (2007). The tool takes as input the skeleton graph and the traffic matrix. For each arc $(u, v)$, it produces an integer OSPF weight $w(u, v)$ in the range $[0,30]$ and an integer capacity $c(u, v)$, such that if all traffic is routed according to the OSPF weights (with traffic splitting if there is more than one shortest path from one vertex to another), no arc will have traffic load exceeding $85 \%$ of its capacity.

It does this while attempting to minimize the total arc cost $\sum_{(u, v)} l(u, v) \cdot c(u, v)$. The optimization is done via a biased random key genetic algorithm, using a population of size 50 and run for 100 iterations. For more details, see Buriol et al. (2007). (Running with larger parameter values would likely produce lower-cost solutions to the design problem, but this would increase the running time, and our goal here is simply to produce a sparse network on which the demand can be routed.)

Note that any arc $(u, v)$ that is on some shortest path for the computed OSPF weights must have nonzero capacity $c(u, v)$, since every traffic demand is nonzero and so every shortest path must carry some traffic. We thus can safely delete all capacity-zero arcs from our network. In order to insure that our graphs remain symmetric, however, we only delete a capacity-zero arc $(a, b)$ if its partner $(b, a)$ also has zero capacity.

\subsubsection{Step 4: Customers and Potential Facility Locations}

For each of our 80 skeleton graphs (10 for each of our eight sets of GT-ITB parameters), we generated seven networks, differing in their choices of the sets $C \subseteq F$ of customer vertices and potential facility locations. The sizes were chosen as a set of fixed fractions of the set $V$ of vertices. Let $(\mathrm{C} x, \mathrm{~F} y)$ denote the set of instances with $|C|=\lceil|V| / x\rceil$ and $|F|=\lceil|V| / y\rceil$. For each graph we generated one instance of each of the following types: (C1,F1), (C2,F2), (C4,F4), (C8,F8), (C2,F1), $(\mathrm{C} 4, \mathrm{~F} 1)$, and $(\mathrm{C} 8, \mathrm{~F} 1)$.

Our synthetic instance testbed thus contains a total of 560 instances, each consisting of a network, OSPF weights (which determine the shortest paths in the network), and the sets $C$ and $F$. The full set of synthetic instances is available from author Resende, and currently can be downloaded from http://mauricio.resende.info/covering-by-pairs/.

\subsection{Real-World ISP-Based Instances}

The 70 real-world instances in our testbed were derived from 10 proprietary Tier-1 Internet Service Provider (ISP) backbone networks and many of them use actual OSPF weights. Because of the proprietary nature of these instances, we will not be able to report as detailed and precise results about them, although we shall summarize how they differ from the synthetic instances, and how the results for the two instance types compare.

The ISP networks ranged in size from a little more than 100 routers to over 5000, each with between $3.5|V|$ and $4.3|V|$ arcs (similar to the range for our synthetic instances). We shall denote them by R100a, R100b, R200, R300, R500, R700, R1000, R1400, R3000, and R5500. where the number following the $\mathrm{R}$ is the number of routers, rounded to the nearest multiple of 100 . (Because of their proprietary nature, these instances cannot be made publicly available.)

For four of these instances (R100a, R100b, R200, and R500), we only had information about the topology, but not the roles of the routers. For each of these, we constructed 16 instances, starting with the case in which $F=C=V$. The other instances also had $F=V$, but $C$ was a random sample of roughly $1 / 2,1 / 4$, or $1 / 8$ of the vertices. Following our notation for the synthetic instances, we shall refer to these four alternatives as $(\mathrm{C} 1, \mathrm{~F} 1),(\mathrm{C} 2, \mathrm{~F} 1),(\mathrm{C} 4, \mathrm{~F} 1)$, and $(\mathrm{C} 8, \mathrm{~F} 1)$. In addition to the single $(\mathrm{C} 1, \mathrm{~F} 1)$ instance, we generated five distinct instances for each alternative from $(\mathrm{C} 1, \mathrm{~F} 2),(\mathrm{C} 1, \mathrm{~F} 4),(\mathrm{C} 1, \mathrm{~F} 8)$, based on different random choices for $C$. 
For R300, R700, R1000, R1400, R3000, and R5500, where we have more detailed information about the roles played by the routers, we constructed instances, one for each topology, that took those roles into account: routers most closely associated with network customers (access routers, etc.) served as the customer vertices, and the potential facility locations consisted of these, together with all other routers except for the backbone routers (thus modeling the likely situation in which extra monitoring equipment cannot easily be connected to the backbone routers). The number of customer vertices ranged between $50 \%$ and $93 \%$ of the total, and the number of vertices that were neither customers nor potential facility locations ranged from $2 \%$ to $41 \%$.

\subsection{Instance Properties}

Our synthetic instances reflect the structure of real-world LANs and WANs, and consist of many, relatively small 2-connected components. For instances with $|V| \geq 100$, the largest 2-connected component averages around $12 \%$ of the vertices (112.5 vertices for our $|V|=984$ instances). In contrast, the Tier-1 ISP instances consist of one very large 2-connected component, typically containing over $95 \%$ of the vertices, with small 2-connected components hanging off of it, most of size 2. One consequence of this difference in topology is differing numbers of triples for the two instance types.

For a given instance with a set $C$ of customers and a set $F$ of potential facility locations, there are $|C|(|F|-1)(|F|-2) / 2$ potential triples $\left(c, f, f^{\prime}\right)$, each one containing a customer $c$ and two distinct potential facility locations in $F-\{c\}$, For our synthetic instances, only a relatively small fraction of the potential triples are actually valid triples in the set-disjoint case. For each class $(C x, F y)$, the percentage of valid triples declines as $N$ increases, until it appears to stabilize somewhere between 4 and 5\%. See Table 2, The ISP-based instances yield substantially more set-disjoint triples than the synthetic ones of the same size. The percentage of valid triples, with one exception, ranges between 20 and 35\%, the exception being R1000, which at $11.2 \%$ still has more than double the percentage for the large synthetic instances. See Table 3 . Note that, because of the cubic growth in the number of potential triples, the actual number of triples can become quite large. For our largest instance, R5500, there are over 5.7 billion valid triples in the set-disjoint case.

The choice of arc weights also has an effect on our instances. The optimized weights of our synthetic instances lead to relatively few shortest-path ties, and so the pathwise-disjoint instances typically have only 5 to $6 \%$ more valid triples than the corresponding set-disjoint instances. The weights in the ISP instances were constrained by a variety of factors in addition to traffic balancing, and yield far more ties. As a result, the number of path-disjoint triples exceeds the number of setdisjoint triples by from 24 to 335\%, that latter occurring for our anomalous R1000 instance.

We also measured the effect of relaxing the constraints on our path-disjoint instances, by requiring only that the paths be arc-disjoint, not vertex-disjoint. (Although vertex-disjointness adds robustness to our monitoring application, the mathematics of the application only requires arcdisjointness.) Here the effects were relatively minor for both synthetic and ISP-based instances, typically yielding an increase of less than $2 \%$ in the number of valid triples. Instance R1000 was again the major exception, yielding an increase of $15 \%$, to reach a total of more than half the maximum possible number of triples.

Finally, to further explore the effect of increased numbers of ties in the set-disjoint case, we considered modified versions of our instances in which all edge weights are set to 1 . As might be expected for such weights, the number of set-disjoint triples drops substantially for almost all our synthetic and ISP-based instances (the only exception again being instance R1000, where we saw a $16 \%$ increase).

\section{Summary of Experimental Results}

This section evaluates our algorithms in three areas.

- Accuracy: How close to optimal were the solutions provided by each of heuristics GreEDy, 
Synthetic Instances

\begin{tabular}{r|rrrrrrrr} 
Class & 50 & 100 & 190 & 220 & 250 & 300 & 558 & 984 \\
\hline \multicolumn{7}{c}{ Average Number of Triples in Thousands } \\
\hline C1,F1 & 12.9 & 57.8 & 224.8 & 340 & 487 & 756 & 4,111 & 19,954 \\
C2,F1 & 6.3 & 31.6 & 119.1 & 184 & 270 & 375 & 2,070 & 10,310 \\
C4,F1 & 3.5 & 15.1 & 53.6 & 92 & 118 & 185 & 1,131 & 5,136 \\
C8,F1 & 2.2 & 7.5 & 28.0 & 44 & 59 & 98 & 471 & 2,642 \\
C2,F2 & 1.5 & 6.7 & 26.9 & 43 & 55 & 93 & 507 & 2,438 \\
C4,F4 & 0.2 & 0.8 & 3.7 & 5 & 8 & 11 & 62 & 299 \\
C8,F8 & 0.0 & 0.1 & 0.4 & 1 & 1 & 1 & 6 & 38 \\
\hline \multicolumn{7}{c}{ Average Percent of Total Number of Possible Triples for Set-Disjoint Instances } \\
\hline C1,F1 & 21.99 & 11.92 & 6.66 & 6.48 & 6.30 & 5.65 & 4.76 & 4.20 \\
C2,F1 & 21.58 & 13.02 & 7.05 & 7.00 & 6.98 & 5.61 & 4.79 & 4.34 \\
C4,F1 & 22.63 & 12.43 & 6.28 & 7.00 & 6.09 & 5.53 & 5.22 & 4.33 \\
C8,F1 & 26.42 & 11.92 & 6.56 & 6.60 & 5.94 & 5.80 & 4.35 & 4.45 \\
C2,F2 & 22.03 & 11.33 & 6.48 & 6.59 & 5.73 & 5.65 & 4.72 & 4.12 \\
C4,F4 & 22.03 & 11.30 & 7.20 & 6.50 & 6.82 & 5.63 & 4.64 & 4.07 \\
C8,F8 & 20.10 & 15.23 & 7.32 & 7.24 & 6.99 & 5.57 & 3.83 & 4.16 \\
\hline Average Percent Increase in Triples for Pathwise-Disjoint Instances over Set-Disjoint Ones \\
\hline C1,F1 & 6.94 & 6.63 & 4.96 & 5.99 & 3.88 & 6.68 & 4.29 & 5.60 \\
C2,F1 & 6.93 & 5.73 & 5.05 & 5.10 & 3.25 & 5.99 & 4.09 & 4.90 \\
C4,F1 & 7.52 & 5.00 & 4.87 & 6.08 & 3.84 & 7.10 & 2.74 & 4.23 \\
C8,F1 & 5.28 & 5.14 & 3.93 & 7.49 & 3.26 & 5.60 & 3.37 & 2.88 \\
C2,F2 & 8.32 & 7.13 & 4.36 & 5.37 & 4.50 & 6.62 & 3.88 & 6.15 \\
C4,F4 & 3.99 & 6.98 & 7.46 & 8.12 & 4.38 & 7.60 & 4.97 & 5.69 \\
C8,F8 & 17.71 & 7.20 & 5.61 & 3.32 & 1.98 & 5.63 & 4.62 & 6.15 \\
\hline \multicolumn{7}{c}{ Average Percent Decrease in Triples for Equal-Weights } & on Set-Disjoint Instances \\
\hline C1,F1 & 16.88 & 13.82 & 9.07 & 10.29 & 14.53 & 13.22 & 26.89 & 31.13 \\
C2,F1 & 15.48 & 15.12 & 10.48 & 9.67 & 15.11 & 14.89 & 26.68 & 31.09 \\
C4,F1 & 11.82 & 15.48 & 9.52 & 9.79 & 14.50 & 14.44 & 30.95 & 32.24 \\
C8,F1 & 22.68 & 8.64 & 4.92 & 7.68 & 12.71 & 15.31 & 24.03 & 39.41 \\
C2,F2 & 13.81 & 14.27 & 4.47 & 13.34 & 11.84 & 11.26 & 26.48 & 30.23 \\
C4,F4 & 13.60 & 13.03 & 7.46 & 9.13 & 20.49 & 3.16 & 24.81 & 32.15 \\
C8,F8 & 8.35 & 17.06 & 5.55 & 19.95 & 14.86 & 4.89 & 27.72 & 37.81 \\
\hline
\end{tabular}

Table 2: Average numbers of triples for synthetic instances, the percentage of all possible triples that these numbers represent, the percentage increases in numbers of triples for the arc-disjoint version of pathwise-disjoint instances, and for equal-weight instances. 
ISP-Based Instances

\begin{tabular}{|c|c|c|c|c|c|c|c|c|c|c|}
\hline Class & R100a & R100b & $\mathrm{R} 200$ & R300 & R500 & R700 & R1000 & $\mathrm{R} 1400$ & R3000 & R5500 \\
\hline \multicolumn{11}{|c|}{ Average Number of Triples in Millions } \\
\hline $\mathrm{C} 1, \mathrm{~F} 1$ & 0.22 & 0.36 & 1.44 & 2.47 & 17.7 & 22.0 & 47.2 & 134 & 816 & 8,156 \\
\hline $\mathrm{C} 2, \mathrm{~F} 1$ & 0.11 & 0.18 & 0.73 & & 8.8 & & & & & \\
\hline $\mathrm{C} 4, \mathrm{~F} 1$ & 0.06 & 0.09 & 0.36 & & 4.4 & & & & & \\
\hline $\mathrm{C} 8, \mathrm{~F} 1$ & 0.03 & 0.05 & 0.20 & & 2.2 & & & & & \\
\hline \multicolumn{11}{|c|}{ Average Percent of Total Number of Possible Triples for Set-Disjoint Instances } \\
\hline $\mathrm{C} 1, \mathrm{~F} 1$ & 30.0 & 34.0 & 25.6 & 33.7 & 23.9 & 28.2 & 11.2 & 21.4 & 35.0 & 20.9 \\
\hline $\mathrm{C} 2, \mathrm{~F} 1$ & 30.1 & 34.1 & 26.0 & & 23.5 & & & & & \\
\hline $\mathrm{C} 4, \mathrm{~F} 1$ & 32.2 & 32.7 & 25.4 & & 23.6 & & & & & \\
\hline $\mathrm{C} 8, \mathrm{~F} 1$ & 26.5 & 32.8 & 27.0 & & 23.3 & & & & & \\
\hline
\end{tabular}

Average Percent Increase in Triples for Pathwise-Disjoint Instances over Set-Disjoint Ones

\begin{tabular}{l|llllllllll} 
C1,F1 & 43.6 & 40.1 & 59.8 & 41.7 & 24.9 & 31.0 & 335.6 & 33.0 & 25.9 & 101.7 \\
C2,F1 & 42.6 & 39.9 & 58.4 & & 25.3 & & & & & \\
C4,F1 & 41.2 & 46.5 & 63.6 & & 26.7 & & & & & \\
C8,F1 & 53.4 & 36.2 & 56.8 & & 24.0 & & & & & \\
\hline
\end{tabular}

Average Percent Increase in Triples for Arc-Disjoint over Vertex Disjoint Instances

\begin{tabular}{|c|c|c|c|c|c|c|c|c|c|c|}
\hline $\mathrm{C} 1, \mathrm{~F} 1$ & 1.0 & 1.3 & 1.3 & .1 & 1.8 & .3 & 15.5 & 1.5 & .1 & 5.5 \\
\hline $\mathrm{C} 2, \mathrm{~F} 1$ & 1.0 & 1.3 & 1.2 & & 1.9 & & & & & \\
\hline $\mathrm{C} 4, \mathrm{~F} 1$ & 1.4 & 1.4 & 1.3 & & 1.9 & & & & & \\
\hline $\mathrm{C} 8, \mathrm{~F} 1$ & .8 & 1.6 & 1.3 & & .9 & & & & & \\
\hline
\end{tabular}

Average Percent of Total Number of Possible Triples for Arc-Path-Disjoint Instances

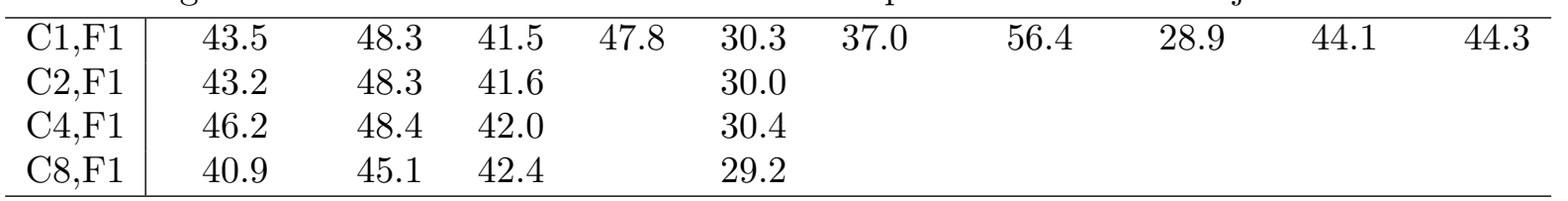

$\begin{array}{cccc}\mathrm{C} 8, \mathrm{~F} 1 & 40.9 & 29.2 & \\ \text { Average Percent Decrease in Triples for Equal-Weights on Set-Disjoint Instances }\end{array}$

\begin{tabular}{|c|c|c|c|c|c|c|c|c|c|c|}
\hline $\mathrm{C} 1, \mathrm{~F} 1$ & 27.4 & 16.0 & 4.9 & 29.4 & 14.2 & 31.6 & -16.7 & 15.7 & 55.1 & 58.7 \\
\hline $\mathrm{C} 2, \mathrm{~F} 1$ & 26.1 & 16.1 & 5.7 & & 13.7 & & & & & \\
\hline $\mathrm{C} 4, \mathrm{~F} 1$ & 27.0 & 11.9 & 6.3 & & 12.0 & & & & & \\
\hline $\mathrm{C} 8, \mathrm{~F} 1$ & 30.1 & 15.3 & 2.7 & & 16.9 & & & & & \\
\hline
\end{tabular}

Table 3: Average percentage of valid triples for real world instances, the percentage increases in numbers of triples for the vertex- and arc-disjoint versions of pathwise-disjoint instances, and for equal-weight instances. 
SHS, and DHS? For small instances, for which the exact optimal can be computed using CPLEX, we can compare to that. In the set-disjoint case, where the SHS lower bound turns out to equal the optimal solution value for all our instances, we can compare to that. For large path-disjoint instances, we have no good, readily computable, standard of comparison. We consider two options. First, we can compare the results to the (very weak but linear-time computable) lower bound obtained when one requires only that paths be vertex-disjoint, not that they be shortest paths. Second, we can evaluate the results for GreEdy(400) in the path-disjoint case by attempting to improve on them using the GENETIC algorithm and large amounts of computing time. (Note that GENETIC optimally solved all the bad set-disjoint instances for GREedy(400) except R5500, which is too big for Genetic to handle.)

- Running Time: How fast are the algorithms? Our runs were primarily performed on two machines. We used a shared-memory machine with $1.5 \mathrm{Ghz}$ Itanium processors and 256 Gigabytes of random access memory, running SUSE LINUX with kernel 2.6.16.60-0.81.2default, for the heuristic runs on instance R5500, where 80 gigabytes of main memory were needed because of the large number of triples involved. In the text this will be referred to as "our slower machine." The remainder of our runs were primarily performed on a shared memory multiprocessor machine with $242.7 \mathrm{Ghz}$ Intel Xeon processors and 50 gigabytes of random access memory running CentOS Linux with kernel 2.6.32-431.11.2.el6.x86_64Linux. In addition, we did some confirmatory runs on a MacBook Pro with a $2.4 \mathrm{Ghz}$ dual-core Intel i5 processor and 8 gigabytes of RAM, running Mac OS 10.9.4 and with Gurobi 5.6 replacing CPLEX 11.0 as our MIP solver. When we report running times, we give the sum of the system and user times, and will specify the machine used. (Each algorithm run used only a single processor/core of the respective machine.) All our codes were written in C, and compiled using gcc. Unless otherwise stated, the running times reported are for code compiled with no optimization flags set, although, where appropriate, we will indicate the kinds of speedups one might expect using -02 optimization.

It should be noted that on none of these machines were running times precisely repeatable. Differences of $20 \%$ or more on duplicate runs were not uncommon. So our running time reports can provide only order-of-magnitude information, and can provide evidence that one code is faster than another only if the differences are substantial and hold across many instances.

- Solution Quality (Cost Reduction): The first two measures address the quality of the algorithms. This one addresses what they tell us about the applications. Even an optimal solution will not be enough to justify the proposed monitoring scheme of $\mathrm{Gu}$ et al. (2008) if it does not provide a significant savings over the default solution that simply takes all customers (which for our instances are themselves facility locations) and is likely to yield more reliable measurements. We also consider how much further improvement is obtainable when going from the setwise-disjoint to the pathwise-disjoint versions of the problem.

\subsection{Accuracy and Running Times for the Set-Disjoint Case}

\subsubsection{Optimization via our MIP Code}

In Table 4, we summarize the results for our MIP optimization code on our synthetic and ISP-based set-disjoint instances. All the runs were performed on the faster of our two Linux machines. The first section of the table reports how many of the 10 instances of each type that the code was able to solve within a 24-hour time bound. For $|V|=190$, we eventually solved the missing $(\mathrm{C} 4, \mathrm{~F} 1)$ and $(\mathrm{C} 8, \mathrm{~F} 1)$ instances, although the longest $(\mathrm{C} 4, \mathrm{~F} 1)$ took slightly more than a week. We also solved the two missing $(\mathrm{C} 4, \mathrm{~F} 1)$ instances based on the ISP-based R200 instances, although the longer took over a week. On the other hand, for 299 of the 383 solved instances, MIP took less than 10 minutes. The second section of the table reports the worst running times (system time plus user time, rounded to the nearest second) for the classes where all instances were solved in less than 24 
Synthetic Instances

ISP-Based Instances

\begin{tabular}{|c|c|c|c|c|c|c|c|c|c|c|c|}
\hline Class & 50 & 100 & 190 & 220 & 250 & 300 & 558 & 984 & R100a & R100b & $\mathrm{R} 200$ \\
\hline \multicolumn{12}{|c|}{ Number of Instances Solved in Less Than 24 Hours by MIP } \\
\hline$(\mathrm{C} 1, \mathrm{~F} 1)$ & 10 & 10 & 10 & - & - & - & - & - & 1 & 1 & 1 \\
\hline$(\mathrm{C} 2, \mathrm{~F} 1)$ & 10 & 10 & 10 & - & - & - & - & - & 5 & 5 & - \\
\hline$(\mathrm{C} 4, \mathrm{~F} 1)$ & 10 & 10 & 9 & - & - & - & - & - & 5 & 5 & 3 \\
\hline (C8,F1) & 10 & 10 & 9 & - & - & - & - & - & 5 & 5 & 5 \\
\hline$(\mathrm{C} 2, \mathrm{~F} 2)$ & 10 & 10 & 10 & 10 & 10 & 10 & - & - & & & \\
\hline$(\mathrm{C} 4, \mathrm{~F} 4)$ & 10 & 10 & 10 & 10 & 10 & 10 & 10 & 10 & & & \\
\hline$(\mathrm{C} 8, \mathrm{~F} 8)$ & 10 & 10 & 10 & 10 & 10 & 10 & 10 & 10 & & & \\
\hline \multicolumn{12}{|c|}{ Worst Running Times (in Seconds) for Instances of Each Completed Class } \\
\hline$(\mathrm{C} 1, \mathrm{~F} 1)$ & 20 & 89 & 23 & 518 & 42,998 & - & - & - & 0 & 0 & 74 \\
\hline$(\mathrm{C} 2, \mathrm{~F} 1)$ & 18 & 86 & 1725 & - & - & - & - & - & 8 & 1 & - \\
\hline$(\mathrm{C} 4, \mathrm{~F} 1)$ & 36 & 46 & - & - & - & - & - & - & 291 & 92 & - \\
\hline$(\mathrm{C} 8, \mathrm{~F} 1)$ & 35 & 803 & - & - & - & - & - & - & 13 & 46 & 33,789 \\
\hline$(\mathrm{C} 2, \mathrm{~F} 2)$ & 0 & 0 & 0 & 0 & 2 & 25 & - & - & & & \\
\hline$(\mathrm{C} 4, \mathrm{~F} 4)$ & 0 & 0 & 0 & 0 & 0 & 0 & 0 & 227 & & & \\
\hline$(\mathrm{C} 8, \mathrm{~F} 8)$ & 0 & 0 & 0 & 0 & 0 & 0 & 0 & 0 & & & \\
\hline
\end{tabular}

Table 4: Results for our MIP optimization code on set-disjoint instances.

hours. For our testbed in general, the code tends to run into trouble as the number of customers grows beyond 100, with exceptions when the number of facilities is also relatively small. Its running times seem clearly to be growing in exponential fashion as $|C|$ increases. Note also that, when all vertices are potential facility locations, reducing the number that are also customers can actually make the instance harder.

\subsubsection{Lower Bounds and Solutions via our HSLB Code}

Fortunately, the exponential behavior of our MIP code is not an insurmountable difficulty in the case of our set-disjoint instances. Not only did our HSLB code produce a lower bound that equals the optimal solution value for all our instances, but the hitting set it constructed was always itself a feasible, and hence optimal, solution. Moreover, it took no more than 4 seconds on any instance besides R5500, for which it only took 22 seconds. In general, it was far faster than any of our heuristics, often by a factor of 100 or more, even though it involves finding an optimal solution to an instance of the NP-hard HitTing SET problem. Moreover, its space requirements are much less than those of our heuristics, given that the (Customer,Neighbor,Facility) triples it stores are far fewer in number than the standard (Customer,Facility,Facility) triples required by the heuristics (and stored by them in triplicate). Consequently, for our largest instance, R5500, it required just over $1 \mathrm{~GB}$, whereas the heuristics need in excess of $65 \mathrm{~GB}$.

\subsubsection{The Heuristics}

As for the heuristics, the "best-of-400-iterations" versions of GREEDY, SHS, and our two variants on DHS also all performed well in general on our test bed, although two instances were seriously problematical for at least one of them.

The best solutions found (over 400 runs) by SHS were optimal for all the instances in our testbeds, both synthetic and ISP-based. DHS $_{H}$ and GREEDY performed almost as well. The former failed to produce an optimal solution for just two instances, on one missing optimal by just a little, but on the other missing by a lot. Its solution for one of our (C1,F1), $|V|=984$ synthetic instances was one facility larger than optimal, but its solution for the ISP-based instance R1000 was off by more than a factor of 3. GREEDY was optimal for all our synthetic instances and all but three 


\begin{tabular}{|c|c|c|c|c|c|c|c|c|}
\hline Algorithm & R200 & R300 & R500 & $\mathrm{R} 700$ & $\mathrm{R} 1000$ & $\mathrm{R} 1400$ & R3000 & $\mathrm{R} 5500^{*}$ \\
\hline \multicolumn{9}{|c|}{ Linux Machine(s), Compiled without Optimization } \\
\hline HSLB & 0.1 & 0.1 & 0.4 & 0.3 & 1 & 1 & 4 & 23 \\
\hline Triple Gen & 0.1 & 0.2 & 0.9 & 1.4 & 4 & 8 & 41 & 1,800 \\
\hline SHS & 7.3 & 4.7 & 68.3 & 32.0 & 125 & 361 & 452 & 23,170 \\
\hline $\mathrm{DHS}_{1}$ & 7.2 & 6.7 & 73.1 & 37.9 & 147 & 389 & 524 & 24,560 \\
\hline $\mathrm{DHS}_{H}$ & 8.4 & 11.6 & 74.1 & 75.8 & 396 & 730 & 1165 & 47,970 \\
\hline GreEDY & 11.8 & 14.0 & 116.1 & 90.9 & 578 & 704 & 2171 & 70,100 \\
\hline \multicolumn{9}{|c|}{ MacBook Pro, Compiled with Optimization } \\
\hline HSLB & 0.8 & 0.7 & 1.2 & 1.0 & 1 & 1 & 3 & 14 \\
\hline Triple Gen & 0.5 & 0.5 & 0.8 & 0.9 & 2 & 3 & 14 & - \\
\hline SHS & 2.2 & 2.0 & 17.7 & 8.8 & 32 & 94 & 179 & - \\
\hline $\mathrm{DHS}_{1}$ & 2.4 & 2.5 & 19.0 & 13.4 & 44 & 113 & 265 & - \\
\hline $\mathrm{DHS}_{H}$ & 2.5 & 2.3 & 19.8 & 14.9 & 130 & 119 & 277 & - \\
\hline GREEDY & 3.2 & 4.1 & 30.3 & 25.9 & 188 & 198 & 866 & - \\
\hline
\end{tabular}

Table 5: Running times in seconds for triple construction, full 400-iteration heuristic runs, and HSLB computations. *For R5500, all Linux times except those for HSLB are on the slower of our two machines, because of memory constraints, and for 80-iteration runs. Memory constraints also prevented running anything except HSLB on the MacBook Pro for this instance.

of our ISP-based instances. It was slightly worse than $\mathrm{DHS}_{H}$ on R1000 and off by a factor of 2.2 on R5500. Its third nonoptimality was minor, consisting of a solution with one more facility than optimal on the second of five $(\mathrm{C} 2, \mathrm{~F} 1)$ variants on R500. Although $\mathrm{DHS}_{1}$ found optimal solutions on all our ISP-based instances, if failed to find optimal solutions for seventeen of our synthetic instances, six of the ones with $|V|=558$ and eleven of those with $|V|=984$, including the one that on which $\mathrm{DHS}_{H}$ failed. On twelve it was off by one facility, on four it was off by two, and on one it was off by five. In no case, however, was the discrepancy greater than $3.3 \%$. The better of the $\mathrm{DHS}_{H}$ and $\mathrm{DHS}_{1}$ solutions was always optimal.

To further compare these heuristics, let us consider two other metrics, running time and robustness, together with the tradeoffs between the two. See Table 5, which reports the running times in seconds for the 400-iteration versions of our heuristics on our faster Linux machine (80-iteration runs on our slower machine for R5500, which would not fit on the faster machine), and on our MacBook Pro, where Gurobi 5.6 replaced CPLEX 11.0 as our MIP solver, and we compiled with the -O2 optimization flag set. The instances covered are the largest ones in our testbed for each ISP topology with $|V| \geq 200$, i.e., the single instance for each topology where we knew the OSPF weights and router types, and the $(\mathrm{C} 1, \mathrm{~F} 1)$ instance for each of the instances where we optimized the weights ourselves. For comparison purposes, the table also breaks out the time devoted to constructing the triples used by the heuristics, and the times for our code that computes the HSLB and determines whether the optimal hitting set produced is a feasible solution. Running times for our synthetic instances of similar sizes were roughly comparable.

The algorithms are typically ranked SHS, DHS ${ }_{1} \mathrm{DHS}_{H}$, GREEDY in order of increased running times for both machines, although there are minor inversions for a few instances. The large differences typically seen between $\mathrm{DHS}_{H}$ and $\mathrm{DHS}_{1}$ on the Linux machines occurred for only one instance (R1000) on the MacBook Pro runs. The fact that, for the smaller instances, the running times for HSLB and triple generation are larger on the optimized MacBook Pro runs than on the unoptimized Linux runs is attributable to larger system times for triple construction. For the (facility,neighbor,facility) triples used in computing the HSLB, the system time was roughly 0.7 seconds, independent of instance size, on the MacBook Pro, versus 0.1 seconds on the Linux machine, and the differences in system times for computing standard triples in our heuristics were similar. It is interesting to note that, on both machines, the growth in user time for triple construction appears to be somewhat slower than our theoretical bound of $\Theta\left(|V|^{3}\right)$, although no firm conclusions can be 


\begin{tabular}{r|ccccc}
$\mathrm{k}$ & $N=100$ & $N=200$ & $N=400$ & $N=800$ & $\mathrm{~N}=1600$ \\
\hline 1 & 0.77855704 & 0.60615106 & 0.36741911 & 0.13499680 & 0.01822414 \\
2 & 0.60577044 & 0.36695782 & 0.13465804 & 0.01813279 & 0.00032880 \\
3 & 0.47103323 & 0.22187230 & 0.04922732 & 0.00242333 & 0.00000587 \\
4 & 0.36603234 & 0.13397967 & 0.01795055 & 0.00032222 & 0.00000010 \\
5 & 0.28425652 & 0.08080177 & 0.00652893 & 0.00004263 & 0.00000000 \\
6 & 0.22060891 & 0.04866829 & 0.00236860 & 0.00000561 & 0.00000000 \\
7 & 0.17110232 & 0.02927600 & 0.00085708 & 0.00000073 & 0.00000000 \\
8 & 0.13261956 & 0.01758795 & 0.00030934 & 0.00000010 & 0.00000000 \\
9 & 0.10272511 & 0.01055245 & 0.00011135 & 0.00000001 & 0.00000000 \\
10 & 0.07951729 & 0.00632300 & 0.00003998 & 0.00000000 & 0.00000000 \\
11 & 0.06151216 & 0.00378375 & 0.00001432 & 0.00000000 & 0.00000000 \\
12 & 0.04755251 & 0.00226124 & 0.00000511 & 0.00000000 & 0.00000000 \\
13 & 0.03673647 & 0.00134957 & 0.00000182 & 0.00000000 & 0.00000000 \\
14 & 0.02836164 & 0.00080438 & 0.00000065 & 0.00000000 & 0.00000000 \\
15 & 0.02188134 & 0.00047879 & 0.00000023 & 0.00000000 & 0.00000000 \\
\hline
\end{tabular}

Table 6: Probabilities of failure to find an optimal solution in $N$ independent runs when the probability of finding one in 400 runs is $k / 400$.

drawn given the differences in topology and weight settings for the various instances.

The general take-away from these results, however, is that all of our algorithms have running times that would be practical in the context of our proposed applications, where the time for implementing a solution will still greatly exceed the time to compute it. The main obstacle to practicality is the memory requirement for instances significantly larger than R3000, and in the final section of this paper, we will discuss ways that this memory requirement might be reduced while keeping running times practical.

Of course, although running time is not a substantial obstacle, it is always worthwhile to find ways to reduce it (beyond simply using optimized compilation). One obvious way would be to perform fewer than 400 iterations. Conversely, we might want give up running time and perform more iterations, if that would improve the robustness of our results. How robust are they?

In the context of our experiments, let $k$ be the number of runs (out of our 400) that returned an optimal solution. We can then roughly estimate the probability of finding an optimal solution to be $k / 400$, and the probability that such a solution will not be found if we perform another $N$ independent random runs is $((400-k) / 400)^{N}$. Table 6 gives the probabilities for $1 \leq k \leq 15$ and $N \in\{100,200,400,800,1600\}$. Using this table, we can evaluate the likely robustness of our heuristics, based on the instances with the smallest values of $k$ for each heuristic. If we want to reduce the failure probability to, say, $0.1 \%$, we would need to have observed a 400 -iteration success count of 2 if we were going to perform 1600 iterations, 4 if 800 iterations, 7 if 400 iterations, 14 if 200 iterations, and more than 15 if 100 iterations (actually, a count of 27 would suffice in this case). Given that the counts given by single 400-iteration runs give noisy estimate of the true probabilities, it might be safer to inflate the above count thresholds in practice.

To help us evaluate our four heuristics in this context, Table 7 gives the 15 smallest success counts for each, both for synthetic and ISP-based instances, together with derived counts for an algorithm $\mathrm{DHS}_{1 H}$, which performs 200 runs each for $\mathrm{DHS}_{1}$ and $\mathrm{DHS}_{H}$, and reports the best solution overall. Note that the entries for the derived heuristic are not simply the averages of the values for $\mathrm{DHS}_{1}$ and $\mathrm{DHS}_{H}$ in the same row of the table, since these may represent counts for different instances - the hardest instances for one of the two need not be the same as those for the other. The counts tend to be higher for our ISP-based instances, since 15 represents over $21 \%$ of our 70 instances of that type, whereas it represents only about $2.4 \%$ of our 630 synthetic instances,

Based on the results in the table, one can conclude that GREEDY is the most robust for synthetic instances, with SHS almost as good. Neither $\mathrm{DHS}_{1}$ nor $\mathrm{DHS}_{H}$ is competitive for such instances, and their combination seems less robust than $\mathrm{DHS}_{H}$, the better of the two, by itself. In contrast, 


\begin{tabular}{r|rrrrr|rrrrr} 
& \multicolumn{7}{|c|}{ SSP-Based Instances } \\
$\mathrm{k}$ & SHS DHS $_{1}$ & DHS $_{H}$ & DHS $_{1 H}$ & GREEDY & SHS & DHS $_{1}$ & DHS $_{H}$ & DHS $_{1 H}$ & GREEDY \\
\hline 1 & 10 & 0 & 0 & 0.0 & 10 & 1 & 3 & 0 & 6.0 & 0 \\
2 & 10 & 0 & 1 & 0.5 & 19 & 25 & 5 & 0 & 8.5 & 0 \\
3 & 13 & 0 & 1 & 1.0 & 29 & 27 & 13 & 0 & 20.5 & 7 \\
4 & 16 & 0 & 4 & 2.0 & 50 & 35 & 23 & 1 & 21.0 & 7 \\
5 & 39 & 0 & 4 & 2.0 & 52 & 68 & 37 & 7 & 26.5 & 29 \\
6 & 47 & 0 & 5 & 2.5 & 55 & 73 & 40 & 14 & 40.0 & 29 \\
7 & 52 & 0 & 5 & 3.5 & 61 & 80 & 44 & 28 & 41.5 & 33 \\
8 & 57 & 0 & 6 & 3.5 & 65 & 91 & 49 & 30 & 44.0 & 35 \\
9 & 57 & 0 & 8 & 4.0 & 66 & 95 & 60 & 44 & 58.0 & 53 \\
10 & 60 & 0 & 8 & 4.0 & 69 & 101 & 67 & 46 & 68.0 & 57 \\
11 & 64 & 0 & 9 & 4.5 & 72 & 110 & 68 & 56 & 79.5 & 66 \\
12 & 65 & 0 & 9 & 5.0 & 74 & 136 & 80 & 68 & 87.0 & 85 \\
13 & 65 & 0 & 10 & 5.0 & 79 & 139 & 100 & 93 & 100.0 & 88 \\
14 & 67 & 0 & 10 & 5.5 & 80 & 163 & 107 & 100 & 100.0 & 89 \\
15 & 67 & 0 & 10 & 6.0 & 82 & 177 & 123 & 100 & 103.5 & 98 \\
\hline
\end{tabular}

Table 7: The 15 smallest numbers of optimal solutions found in 400 independent runs over synthetic and ISP-based instances.

for the ISP-based instances, the combination is distinctly more robust than either $\mathrm{DHS}_{1}$ or $\mathrm{DHS}_{H}$ alone, and also dominates GREEDY, although SHS is the best overall, having only one count less than 25. The corresponding instance, where SHS found an optimal solution only once in 400 times, is troublesome, however. This suggests performing a hybrid of SHS with one of our other three heuristics (again running each 200 times and taking the best). If we blend with GREEDY, the smallest counts are 61 (synthetic) and 6.0 (ISP-based). Blending with $\mathrm{DHS}_{1}$ yields corresponding smallest counts of 34.5 and 9.0, and blending with $\mathrm{DHS}_{H}$ yields smallest counts of 46.5 and 10.0. The last choice would seem to be the best overall, as 400 total iterations should likely be enough to keep the failure rate for all instances less than $0.1 \%$, and, depending on the accuracy of our sample, 200 iterations might suffice. All three blends, however, offer significant improvements in robustness over any of the individual heuristics.

\subsubsection{Challenging HSLB: Equal-Weight Edges}

Given the fact that our HSLB code produced hitting sets that were optimal solutions for all 700 instances in our set-disjoint testbed, one might wonder why we should bother with heuristics at all for the set-disjoint case. Perhaps instances where the HSLB is smaller than the optimal solution value, or where the HSLB solution is otherwise infeasible, simply do not arise in practice, possibly because of the restricted topologies in real world LAN/WANs and ISP networks, or because edge weights are optimized to manage traffic. To investigate the latter possibility, we repeated our experiments with all the weights set to 1 - not a very good choice for traffic management, but certainly a natural default, and one that yields a major change in the set of triples in the derived "cover-by-pairs" instances, as observed in Section 6.3.

With these instances, the hitting sets constructed by our HSLB code were still legal and hence optimal covers for all 70 of our ISP-based instances. However, for 51 of our 630 synthetic instances, the hitting set was not a legal cover, and for nine of these, the best solution found by our 400iteration SHS run was still larger than the computed lower bound (although never by more than $2.1 \%)$. These were all $(\mathrm{C} 1, \mathrm{~F} 1)$ instances, five with $|V|=984$, three with $|V|=558$, and one with $|V|=250$, and in all nine cases, the HSLB nevertheless continued to equal the optimal solution size. The optimal solutions for these nine were not found by any of our 400-iteration runs of $\mathrm{DHS}_{1}$ or $\mathrm{DHS}_{H}$. As in our experiments on the synthetic instances in our standard testbed, it was GREEDY that proved to have the best synergies with SHS. Our 400-iteration runs of GREEDY 
Synthetic Instances

ISP-Based Instances

\begin{tabular}{|c|c|c|c|c|c|c|c|c|c|c|c|}
\hline Class & 50 & 100 & 190 & 220 & 250 & 300 & 558 & 984 & R100a & R100b & $\overline{\mathrm{R} 200}$ \\
\hline \multicolumn{12}{|c|}{ Number of Instances Solved in Less Than 24 Hours by MIP } \\
\hline$(\mathrm{C} 1, \mathrm{~F} 1)$ & 10 & 10 & 10 & 10 & 10 & - & - & - & 1 & 1 & 1 \\
\hline$(\mathrm{C} 2, \mathrm{~F} 1)$ & 10 & 10 & 9 & - & - & - & - & - & 5 & 5 & - \\
\hline$(\mathrm{C} 4, \mathrm{~F} 1)$ & 10 & 10 & 8 & - & - & - & - & - & 5 & 5 & 3 \\
\hline$(\mathrm{C} 8, \mathrm{~F} 1)$ & 10 & 10 & 9 & - & - & - & - & - & 5 & 5 & 5 \\
\hline$(\mathrm{C} 2, \mathrm{~F} 2)$ & 10 & 10 & 10 & 10 & 10 & 10 & - & - & & & \\
\hline$(\mathrm{C} 4, \mathrm{~F} 4)$ & 10 & 10 & 10 & 10 & 10 & 10 & 10 & 10 & & & \\
\hline$(\mathrm{C} 8, \mathrm{~F} 8)$ & 10 & 10 & 10 & 10 & 10 & 10 & 10 & 10 & & & \\
\hline \multicolumn{12}{|c|}{ Worst Running Times (in Seconds) for Instances of Each Completed Class } \\
\hline$(\mathrm{C} 1, \mathrm{~F} 1)$ & 21 & 9 & 28 & 19,362 & 78,660 & - & - & - & 0 & 0 & 146 \\
\hline$(\mathrm{C} 2, \mathrm{~F} 1)$ & 18 & 212 & . & - & - & - & - & - & 17 & 21 & - \\
\hline$(\mathrm{C} 4, \mathrm{~F} 1)$ & 35 & 818 & - & - & - & - & - & - & 335 & 474 & - \\
\hline$(\mathrm{C} 8, \mathrm{~F} 1)$ & 25 & 321 & - & - & - & - & - & - & 50 & 644 & 40,739 \\
\hline$(\mathrm{C} 2, \mathrm{~F} 2)$ & 0 & 0 & 0 & 0 & 4 & 13 & - & - & & & \\
\hline$(\mathrm{C} 4, \mathrm{~F} 4)$ & 0 & 0 & 0 & 0 & 0 & 0 & 0 & 8106 & & & \\
\hline$(\mathrm{C} 8, \mathrm{~F} 8)$ & 0 & 0 & 0 & 0 & 0 & 0 & 0 & 0 & & & \\
\hline
\end{tabular}

Table 8: Results for our MIP optimization code on vertex-disjoint instances.

found solutions matching the HSLB for three of the 984's, for one of the 558's, and for the 250 . For the remaining four instances, we needed to increase the number of iterations. For the two remaining 984's, 4000-iteration runs of SHS found solutions matching the HSLB. For one of the two remaining 558's, a 100,000-iteration run of GREEDY found a lower-bound matching solution (three times), although a 200,000-iteration of SHS failed to find any. For the other remaining 558 , a 100,000-iteration run of SHS found a lower-bound matching solution (twice), although a 200,000-iteration run of GREEDY failed to find any.

Thus the track record of HSLB equaling the optimal solution value for "real world" WAN and ISP topologies remains unblemished. We can conclude, however, that we should not count on our HSLB code always producing feasible optimal solutions, and that SHS and GreEDY remain valuable, and complementary, backups.

\subsection{Accuracy and Running Times for the Path-Disjoint Case}

\subsubsection{Optimization via our MIP Code}

Table 8 summarizes our optimization results for the path-disjoint versions of our instances, and shares the format of Table 4, which covered the set-disjoint versions. Once again, we report the number of instances of each class that were solved to optimality within 24 hours, and, for each class with all instances so solved, the worst running time encountered, as a measure of robustness. Note that we were able to solve about the same number of instances of each type within the 24-hour time bound as we did in the set-disjoint case, with the worst time for each type usually slightly worse. In more extended runs, we were able to compute the optimum for the one missing $|V|=190$, (C2,F1) synthetic instance (in 39 hours), and the two missing R200, (C4,F1) ISP-based instances (in 49 and 77 hours).

The results for the arc-disjoint versions of our path-disjoint instances were roughly the same as reported in Table 8 for the vertex-disjoint versions. We were able to solve essentially the same types of instances as before, with times that were roughly equivalent. The maximum for each class tended to be shorter for the arc-disjoint versions, although not so often as to provide a statistically meaningful distinction. 


\begin{tabular}{r|rrrrrrrr} 
Algorithm & R200 & R300 & R500 & R700 & R1000 & R1400 & R3000 & R5500* \\
\hline Linux Machine(s), Compiled without & Optimization & & \\
\hline Path-disjoint Triple Gen & 1.0 & 1.2 & 6.9 & 8.2 & 56 & 72 & 558 & 17,400 \\
Arc-disjoint Triple Gen & 0.7 & 0.6 & 4.9 & 5.4 & 33 & 39 & 262 & 11,200 \\
Set-disjoint Triple Gen & 0.1 & 0.2 & 0.9 & 1.4 & 4 & 8 & 41 & 1,800 \\
\hline Path-disjoint GreEDY(400) & 19.7 & 22.6 & 154.8 & 130.3 & 715 & 664 & 3612 & 79,000 \\
Arc-disjoint GrEEDY(400) & 20.3 & 20.3 & 154.3 & 96.1 & 767 & 548 & 2801 & 68,500 \\
Set-disjoint GreEDy(400) & 11.8 & 14.0 & 116.1 & 90.9 & 578 & 704 & 2171 & 70,100 \\
\hline MacBook Pro, Compiled with Optimization & & & \\
\hline Path-disjoint Triple Gen & 0.7 & 0.7 & 2.7 & 3.5 & 17 & 24 & 347 & \\
Arc-disjoint Triple Gen & 0.6 & 0.6 & 1.5 & 1.8 & 9 & 10 & 95 & - \\
Set-disjoint Triple Gen & 0.5 & 0.5 & 0.8 & 0.9 & 2 & 3 & 14 & - \\
\hline Path-disjoint GreEDy(400) & 4.6 & 5.4 & 38.3 & 35.8 & 239 & 224 & 1865 & - \\
Arc-disjoint GreEDy(400) & 4.4 & 5.2 & 36.3 & 32.7 & 254 & 198 & 1360 & - \\
Set-disjoint GreEDy(400) & 3.2 & 4.1 & 30.3 & 25.9 & 188 & 198 & 866 & - \\
\hline
\end{tabular}

Table 9: Running times in seconds for triple construction and GREedy(400) runs on path-disjoint instances (both vertex- and arc-disjoint versions), with results for set-disjoint instances included for comparison. *For R5500, all Linux times except those for HSLB are on the slower of our two machines, because of memory constraints, and for 80-iteration runs.

\subsubsection{Heuristic Results}

In evaluating heuristics for the path-disjoint case, we suffer from several limitations in comparison to the set-disjoint case. First, the HSLB no longer applies, so we do not have a good standard of comparison for those instances that our MIP approach failed to solve. Second, we cannot use SHS or either of our DHS heuristics, which only apply to the set-disjoint case, and so only have Greedy(400) and its higher-iteration variants (including Genetic) to consider.

Fortunately, GreEDy(400) seems to do very well. It finds the optimal solution on all the instances for which that is known, and we have not been able to find any better solutions than the ones it provides on any of the instances for which the optimum is not known. In particular, we performed 1000-generation runs of our GENETIC algorithm, on each of these instances, and only matched the GREEDY(400) solutions on each.

We had hopes that, if anything could improve on Greedy(400), then Genetic would, based on results we obtained in the set-disjoint case. There, GEnEtic found optimal solutions for two of the three ISP-based instances where GREEDY(400) failed to find the optimum (the third was R5500, which was far too large for Genetic to handle). Most impressively, for R1000 it found the optimum, whereas the best of even 100,000 independent runs of GREEDY was over three times optimal. The failure of GEnETIC to find any improvements in the path-disjoint case thus at least suggests that our GREEDY(400) path-disjoint results might indeed be optimal across the board.

The results were also robust. For each of our 720 synthetic instances, the best solution found was found at least 20 times out of 400. For each of our 69 ISP-based instances other than R5500, the best solution found was found at least four times out of 400 (and for all but one, the count was 34 or greater). The best solution for R5500 was found 72 times out of 80 .

For the arc-disjoint versions of our path-disjoint instances, the results were again similar, both as to accuracy and robustness. For every instance where the optimum was known, GreEDy(400) found it. For all 720 synthetic instances, the best solution found was found at least 34 times out of 400. For all 69 ISP-bases instances other than R5500, the best solution found was found at least six times out of 400 (and for all but one, the count was 41 or greater). The instance with the low count was the same for both vertex- and arc-disjoint versions of the problem. The best solution for R5500 was found 79 times out of 80 .

As to running times, see Table 9, which mimics Table 5 in covering the maximum-customer 
versions of our ISP-based instances with more than 200 vertices, and both the times for triplegeneration and the overall GreEDY(400) times. As before, we present running times for unoptimized code on our Linux machines and optimized code on our MacBook Pro. We list times for both the vertex- and arc-disjoint versions of our path-disjoint instances, and, for comparison purposes, copy from Table 5 the corresponding times for our set-disjoint instances.

Recalling from Table 3 that the number of triples associated with an instances increases as one goes from set-disjoint to vertex-disjoint to arc-disjoint instances, one might expect running times to behave similarly, but this is not the case. For triple generation, the arc-disjoint triples take less time to generate than the vertex-disjoint ones. The explanation for relative triple-generation running times is algorithmic. As described in Section 5.5, the path-disjoint constructions should take longer than the set-disjoint ones because they need an extra network-flow step, and the vertex-disjoint constructions take longer than the arc-disjoint ones because they need to add an extra edge for each vertex in order to insure vertex-disjointness as well as arc-disjointness. Note, however, that, as with the set-disjoint case, the time for vertex- and arc-disjoint triple generation again may well be growing at slightly less than the cubic rate of our worst-case analysis.

Our overall running times also do not precisely track triple counts The times for the pathdisjoint versions of the instances are indeed significantly slower than for the set-disjoint versions (except in the case of R1400), but once again the arc-disjoint times tend to be faster than those for the vertex-disjoint versions. Further study will be needed to understand these discrepancies.

\subsection{Cost Reduction}

In this section we consider the savings our heuristics can provide over simply choosing the cover consisting of the set $C$ of all customer vertices, which is always a feasible solution, and would be the default solution for network path monitoring if we did not use our proposed monitoring scheme.

We first consider the results for our synthetic instances. Table 10 presents our results for the seven classes of instances for each value of $|V| \in\{984,558,300,190,100\}$. (For space reasons, we omit the results for $|V| \in\{250,220,50\}$, but they do not evince any strikingly different behavior.) For each algorithm and instance class, we give both the average percentage of customers that are in the covers produced by the algorithm, and (in parentheses) the percentage reduction over the results in the column to the left. Here "GrEeDY" stands for GreEDY(400), the algorithm that takes the best of 400 independent runs of GREEDY. "Opt" stands for the cover produced by our HSLB code for set-disjoint instances, which for these instances was always optimal. "DP" stands for the optimum when we do not require our vertex-disjoint paths to be shortest paths, as computed by the linear-time dynamic programming algorithm described in Section 3.2. This value is not relevant to either of our applications, but does provide a lower bound on what is possible in the path-disjoint case.

These results clearly show that using our scheme for disjoint path monitoring offers a substantial reduction in the number of vertices needed to cover all the customers. In the set-disjoint case, the reduction is typically by a factor of 4 or more for the $(\mathrm{C} 1, \mathrm{~F} 1)$ instances, in which all vertices are customers, and grows to a factor of 6 when $|V| \in\{558,984\}$. As the proportion of vertices that are customers is reduced for a given value of $|V|$, the savings decreases, but is always more than $29 \%$. Perhaps not surprisingly, when not all vertices are customers, the reduction is greater in the $(\mathrm{C} k, \mathrm{~F} 1)$ classes, where all vertices are potential facility locations, than in the $(\mathrm{C} k, \mathrm{~F} k)$ classes, where the only potential facility locations are the customers themselves. Note that, as remarked before, Greedy(400) finds optimal set-disjoint solutions in all cases, so we omit the parenthetical "percentage reduction" column for OpT.

Going from the set-disjoint to the path-disjoint instances requiring vertex-disjointness typically offers a significant reduction (up to $7 \%$ in the $(\mathrm{C} 1, \mathrm{~F} 1)$ classes with $|V| \in\{558,984\}$ ), although the percentage reduction declines with $|V|$ and $|C|$. Only requiring arc-disjoint paths often yields a small additional improvement, although typically less than $1 \%$. Allowing unbounded vertex-disjoint paths would allow a much more significant reduction, up to almost $40 \%$ in one case, and typically more for the $(\mathrm{C} k, \mathrm{~F} k)$ classes, $k \geq 2$, than in the corresponding $(\mathrm{C} k, \mathrm{~F} 1)$ classes, although the actual 


\begin{tabular}{|c|c|c|c|c|c|c|c|c|c|c|}
\hline \multirow[b]{2}{*}{$|V|$} & \multirow[b]{2}{*}{ Class } & \multirow[b]{2}{*}{$|C|$} & \multicolumn{2}{|c|}{ Set-Disjoint } & \multicolumn{6}{|c|}{ Path-Disjoint } \\
\hline & & & GREeDY & \multirow{2}{*}{$\begin{array}{l}\text { OPT } \\
16.2\end{array}$} & \multicolumn{2}{|c|}{$\begin{array}{c}\text { Vertex-Disjoint } \\
\text { GREEDY }\end{array}$} & \multicolumn{2}{|c|}{$\begin{array}{c}\text { Arc-Disjoint } \\
\text { GrEEDY }\end{array}$} & \multicolumn{2}{|c|}{$\begin{array}{l}\text { Unbounded } \\
\text { DP }\end{array}$} \\
\hline \multirow{7}{*}{984} & (C1,F1) & 984 & $16.2(83.8)$ & & 15.1 & $(7.1)$ & 15.1 & $(0.0)$ & 10.7 & $(29.2)$ \\
\hline & $(\mathrm{C} 2, \mathrm{~F} 1)$ & 492 & $24.0 \quad(76.0)$ & 24.0 & 23.2 & $(3.6)$ & 23.1 & $(0.4)$ & 19.1 & $(17.1)$ \\
\hline & $(\mathrm{C} 2, \mathrm{~F} 2)$ & 492 & $25.7 \quad(74.3)$ & 25.7 & 23.9 & $(6.9)$ & 23.9 & $(0.2)$ & 19.0 & $(20.6)$ \\
\hline & $(\mathrm{C} 4, \mathrm{~F} 1)$ & 246 & $37.9 \quad(62.1)$ & 37.9 & 37.5 & $(1.1)$ & 37.4 & $(0.3)$ & 34.2 & $(8.5)$ \\
\hline & $(\mathrm{C} 4, \mathrm{~F} 4)$ & 246 & $40.1 \quad(59.9)$ & 40.1 & 38.4 & $(4.4)$ & 38.3 & $(0.2)$ & 33.3 & (13.0) \\
\hline & $(\mathrm{C} 8, \mathrm{~F} 1)$ & 123 & $55.4 \quad(44.6)$ & 55.4 & 55.1 & $(0.6)$ & 55.1 & $(0.0)$ & 52.3 & $(5.2)$ \\
\hline & $(\mathrm{C} 8, \mathrm{~F} 8)$ & 123 & $58.2(41.8)$ & 58.2 & 57.2 & $(1.8)$ & 57.1 & $(0.1)$ & 51.2 & $(10.3)$ \\
\hline \multirow{7}{*}{558} & $(\mathrm{C} 1, \mathrm{~F} 1)$ & 558 & $16.0(84.0)$ & 16.0 & 14.9 & $(7.3)$ & 14.9 & $(0.0)$ & 10.7 & $(28.3)$ \\
\hline & $(\mathrm{C} 2, \mathrm{~F} 1)$ & 279 & $24.6 \quad(75.4)$ & 24.6 & 23.6 & $(4.2)$ & 23.5 & $(0.2)$ & 19.6 & $(16.7)$ \\
\hline & $(\mathrm{C} 2, \mathrm{~F} 2)$ & 279 & $25.8 \quad(74.2)$ & 25.8 & 24.5 & $(5.3)$ & 24.4 & $(0.1)$ & 19.2 & $(21.4)$ \\
\hline & $(\mathrm{C} 4, \mathrm{~F} 1)$ & 140 & $37.8 \quad(62.2)$ & 37.8 & 37.1 & $(1.7)$ & 37.1 & $(0.0)$ & 34.4 & $(7.3)$ \\
\hline & $(\mathrm{C} 4, \mathrm{~F} 4)$ & 140 & $42.1 \quad(57.9)$ & 42.1 & 41.2 & $(2.2)$ & 41.1 & $(0.3)$ & 34.6 & $(15.7))$ \\
\hline & $(\mathrm{C} 8, \mathrm{~F} 1)$ & 70 & $56.3(43.7)$ & 56.3 & 56.1 & $(0.3)$ & 56.1 & $(0.0)$ & 53.0 & $(5.6)$ \\
\hline & $(\mathrm{C} 8, \mathrm{~F} 8)$ & 70 & $60.0(40.0)$ & 60.0 & 59.1 & $(1.4)$ & 58.7 & $(0.7)$ & 54.7 & ( 6.8$)$ \\
\hline \multirow{7}{*}{300} & $(\mathrm{C} 1, \mathrm{~F} 1)$ & 300 & $24.0 \quad(76.0)$ & 24.0 & 23.0 & $(4.2)$ & 23.0 & $(0.0)$ & 14.9 & $(35.5)$ \\
\hline & $(\mathrm{C} 2, \mathrm{~F} 1)$ & 150 & $33.7 \quad(66.3)$ & 33.7 & 32.6 & $(3.2)$ & 32.6 & $(0.0)$ & 25.2 & $(22.7)$ \\
\hline & $(\mathrm{C} 2, \mathrm{~F} 2)$ & 150 & $36.1 \quad(63.9)$ & 36.1 & 34.8 & $(3.5)$ & 34.8 & $(0.0)$ & 24.7 & $(29.1)$ \\
\hline & $(\mathrm{C} 4, \mathrm{~F} 1)$ & 75 & $49.1 \quad(50.9)$ & 49.1 & 47.7 & $(2.7)$ & 47.7 & $(0.0)$ & 41.1 & $(14.0)$ \\
\hline & $(\mathrm{C} 4, \mathrm{~F} 4)$ & 75 & $50.8 \quad(49.2)$ & 50.8 & 50.0 & $(1.6)$ & 49.9 & $(0.3)$ & 40.5 & $(18.7)$ \\
\hline & $(\mathrm{C} 8, \mathrm{~F} 1)$ & 38 & $64.5 \quad(35.5)$ & 64.5 & 63.7 & $(1.2)$ & 63.7 & $(0.0)$ & 58.4 & $(8.3)$ \\
\hline & $(\mathrm{C} 8, \mathrm{~F} 8)$ & 38 & $68.4 \quad(31.6)$ & 68.4 & 68.4 & $(0.0)$ & 68.4 & $(0.0)$ & 57.4 & $(16.2)$ \\
\hline \multirow{7}{*}{190} & $(\mathrm{C} 1, \mathrm{~F} 1)$ & 190 & $28.7 \quad(71$ & 28.7 & 27.7 & 10 & 27.7 & ) & 21.0 & $(24.3)$ \\
\hline & $(\mathrm{C} 2, \mathrm{~F} 1)$ & 95 & $40.3(59.7)$ & 40.3 & 39.6 & $(1.8)$ & 39.6 & $(0.0)$ & 33.4 & $(15.7)$ \\
\hline & $(\mathrm{C} 2, \mathrm{~F} 2)$ & 95 & $41.7 \quad(58.3)$ & 41.7 & 40.2 & $(3.5)$ & 40.1 & $(0.3)$ & 33.2 & $(17.3)$ \\
\hline & $(\mathrm{C} 4, \mathrm{~F} 1)$ & 48 & $55.8 \quad(44.2)$ & 55.8 & 55.2 & $(1.1)$ & 55.2 & $(0.0)$ & 51.7 & $(6.4)$ \\
\hline & $(\mathrm{C} 4, \mathrm{~F} 4)$ & 48 & $55.4(44.6)$ & 55.4 & 54.4 & $(1.9)$ & 54.4 & $(0.0)$ & 47.7 & $(12.3)$ \\
\hline & $(\mathrm{C} 8, \mathrm{~F} 1)$ & 24 & $67.1 \quad(32.9)$ & 67.1 & 66.7 & $(0.6)$ & 66.7 & $(0.0)$ & 60.4 & $(9.4)$ \\
\hline & $(\mathrm{C} 8, \mathrm{~F} 8)$ & 24 & $70.8 \quad(29.2)$ & 70.8 & 70.8 & $(0.0)$ & 70.8 & $(0.0)$ & 61.2 & $(13.5)$ \\
\hline \multirow{7}{*}{100} & $(\mathrm{C} 1, \mathrm{~F} 1)$ & 100 & 24.1 & 24.1 & 23.6 & 12 & 23.6 & $(0.0)$ & 14.3 & $(39.4)$ \\
\hline & $(\mathrm{C} 2, \mathrm{~F} 1)$ & 50 & $34.2(65.8)$ & 34.2 & 34.0 & $(0.6)$ & 34.0 & $(0.0)$ & 23.4 & $(31.2)$ \\
\hline & $(\mathrm{C} 2, \mathrm{~F} 2)$ & 50 & $35.4 \quad(64.6)$ & 35.4 & 34.4 & $(2.8)$ & 34.4 & $(0.0)$ & 22.6 & $(34.3)$ \\
\hline & $(\mathrm{C} 4, \mathrm{~F} 1)$ & 25 & $46.8 \quad(53.2)$ & 46.8 & 46.4 & $(0.9)$ & 46.4 & $(0.0)$ & 38.4 & $(17.2)$ \\
\hline & $(\mathrm{C} 4, \mathrm{~F} 4)$ & 25 & $49.2 \quad(50.8)$ & 49.2 & 48.4 & $(1.6)$ & 48.4 & $(0.0)$ & 37.2 & $(23.1)$ \\
\hline & $(\mathrm{C} 8, \mathrm{~F} 1)$ & 13 & $61.5(38.5)$ & 61.5 & 61.5 & $(0.0)$ & 61.5 & $(0.0)$ & 48.5 & $(21.3)$ \\
\hline & $(\mathrm{C} 8, \mathrm{~F} 8)$ & 13 & $63.8(36.2)$ & 63.8 & 62.3 & $(2.4)$ & 61.5 & $(1.2)$ & 47.7 & $(22.5)$ \\
\hline
\end{tabular}

Table 10: Average percentages of customer vertices in algorithmically generated covers for all seven classes of our $|V| \in\{984,558,300,190,100\}$ synthetic instances. Entries in parentheses are the percentage reduction in cover size from the results in the preceding column. 


\begin{tabular}{|c|c|c|c|c|c|c|c|c|c|c|c|}
\hline \multirow[b]{2}{*}{ Instance } & \multirow[b]{2}{*}{$\begin{array}{c}\text { Approx } \\
|C|\end{array}$} & \multicolumn{4}{|c|}{ Set-Disjoint } & \multicolumn{6}{|c|}{ Path-Disjoint } \\
\hline & & \multicolumn{2}{|c|}{ GREEDY } & \multicolumn{2}{|c|}{ OPT } & \multicolumn{2}{|c|}{$\begin{array}{c}\text { Vertex-Disjoint } \\
\text { GREEDY }\end{array}$} & \multicolumn{2}{|c|}{$\begin{array}{c}\text { Arc-Disjoint } \\
\text { GrEEDY }\end{array}$} & \multicolumn{2}{|c|}{$\begin{array}{l}\text { Unbounded } \\
\text { DP }\end{array}$} \\
\hline R5500 & 3200 & 37.7 & $(62.3)$ & 17.0 & $(55.0)$ & 2.8 & $(83.5)$ & 2.8 & $(0.0)$ & 0.6 & $(80.0)$ \\
\hline R3000 & 1500 & 1.8 & $(98.2)$ & 1.8 & $(0$ & 1. & $(25.9)$ & 1.3 & $(0.0)$ & 0.1 & $(90.0)$ \\
\hline $\mathrm{R} 1400$ & 900 & 14.3 & $(85.7)$ & 14.3 & $0.0)$ & 3.2 & $(77.6)$ & 3.2 & $(0.0)$ & 0.3 & $(90.0)$ \\
\hline R1000 & 900 & 35.5 & $(64.5)$ & 10.1 & $(71.5)$ & 3.8 & $(62.0)$ & 3.8 & $(0.0)$ & 0.9 & $(77.1)$ \\
\hline $\mathrm{R} 700$ & 500 & 4.9 & $(95.1)$ & 4.9 & $(0.0)$ & 2.8 & $(43.5)$ & 2.8 & $(0.0)$ & 0.6 & $(76.9)$ \\
\hline $\mathrm{R} 500$ & 500 & 13.9 & $(86.1)$ & 13.9 & $(0$. & 12.4 & $(10.8)$ & 12.4 & $(0.0)$ & 1.9 & $(84.8)$ \\
\hline R300 & 200 & 4.1 & $(95.9)$ & 4.1 & $(0.0)$ & 3.6 & $(11.1)$ & 3.6 & $(0.0)$ & 2.7 & $(25.0)$ \\
\hline $\mathrm{R} 200$ & 200 & 7.6 & $(92.4)$ & 7.6 & $(0.0)$ & 6.7 & $(11.8)$ & 6.7 & $(0.0)$ & 2.2 & $(66.7)$ \\
\hline R100a & 100 & 10.9 & $(89.1)$ & 10.9 & $(0.0)$ & 7.8 & $(28.6)$ & 7.8 & $(0.0)$ & 4.7 & $(40.0)$ \\
\hline $\mathrm{R} 100 \mathrm{~b}$ & 100 & 10.9 & $(89.1)$ & 10.9 & $(0.0)$ & 7.8 & $(28.6)$ & 7.8 & $(0.0)$ & 4.7 & $(40.0)$ \\
\hline
\end{tabular}

Table 11: Average percentages of customer vertices in algorithmically generated covers for our 10 ISP-based instances. Entries in parentheses are the percentage reduction in cover size from the results in the preceding column.

sizes of the covers still remain lower in the latter case.

The results for our ISP-based instances are presented in Table 11, where we only include the $(\mathrm{C} 1, \mathrm{~F} 1)$ versions of those instances where we did not know the types of routers and so could not deduce what the actual sets $F$ and $C$ should be. The resulting value $|C| /|V|=1$ is reasonably close to the corresponding values for the instances where we can estimate the actual sets $F$ and $C$; for these the ratio ranges from about 0.6 to 0.9. Because the instances where we $d o$ have this information are proprietary, the column labeled by " $|C|$ " only lists the value of $C$ rounded to the nearest 100. The average cover sizes reported, however, are given as percentages of the true value of $|C|$. Note that in this table we do include a parenthetical improvement column for Opt over GREEDY(400), since here there are improvements for two of the instances.

As we can see, the effectiveness of our disjoint path monitoring scheme is even more substantial for these instances than for our synthetic ones. The best point of comparison is to the $(\mathrm{C} 1, \mathrm{~F} 1)$ variants of the latter, where the smallest ratio of set-disjoint cover size to $|C|$ was roughly $16 \%$. Here the largest percentage is $17 \%$, and four of the ten are less than $10 \%$, with the percentage for R3000 being just 1.8\%. Moreover, going to the path-disjoint case now offers major improvements, in comparison to the at-most $7.3 \%$ improvement for our synthetic instances. Here the range is from $10.8 \%$ to $83.5 \%$, and now all but one of our instances have covers with size less than $8 \%$ of $|C|$.

Allowing arc-disjoint paths offers no further improvement. However, if one could consider arbitrarily long vertex-disjoint paths, even more substantial reductions are possible. Now half the instances have covers with size less than $1 \%$ of $|C|$. This should not be a surprise, however. As we already observed in Section 6.3, typically $95 \%$ or more of the vertices in our ISP-based instances are in a single large 2-connected component. By the definition of 2-connectedness, any two vertices in such a component will constitute a cover. Additional vertices are only needed to handle the few additional small components hanging off the large one.

\section{Further Results and Directions}

The problems described here can be generalized in a variety of directions, for two of which we already have some preliminary results.

1. Variants of SDFL and PDFL in which we omit the requirement that every customer vertex also be a potential facility location (i.e., that $C \subseteq F$ ). In our monitoring application, it might well be the case that some of the customer vertices are housed in locations that do not have enough free physical space to house our monitoring equipment, or are in leased space into which we do not have permission to add additional equipment. 
2. Variants in which for each vertex a given nonnegative cost must be paid if that vertex is used as a facility, and our goal is to minimize total cost. Note that the second variant includes the first as a special case, since we can simply set the cost of each member of $F$ to be 1 and the cost of all other vertices to be $|F|+1$.

Most of our algorithms and lower bounds carry over more-or-less directly to both types of generalization, with the two exceptions being the linear-time algorithms of Section 5 and Section 4.3 for computing the optimum covers in the special case of trees and our general lower bound for the path-disjoint case. Linear-time algorithms still exist for both of these tasks, but the simple combinatorial characterizations that previously sufficed must now be replaced by dynamic programs of some complexity. Our other algorithms and lower bounds carry over with almost no change for the first type of generalization. For the second type, the integer programs for computing the optimum and for computing the Hitting Set lower bound must change their objective functions so that the variables $x_{f}$ are multiplied by the corresponding costs. And the various cover-constructing heuristics must modify their greedy choices by taking costs into account, for instance by choosing the $f \in F$ which yields the highest ratio (increase in coverage)/(cost of $f$ ).

One question that arises for the first type of variant that was not an issue in our original version of the problems is the question of feasibility. In the original version, where $C \subseteq F$, there was always a feasible cover, mainly the set $C$ itself, with each customer covering itself. When not all customers are potential facility locations, it may be that no cover exists. Thus the first question one must ask is whether such a cover exists. This will be true if and only if the full set $F$ is a valid cover, a property that can easily be checked in $O\left(n^{2} m\right)$ tine by generating the corresponding SCP problem and verifying that there is a valid triple for each customer.

We leave the empirical testing of our algorithms for these generalizations of SDFL and PDFL for further research. At present the variety of potential instance classes is too large for any one or two choices to provide meaningful insights, although future research into potential applications may identify particular instance classes whose practical relevance justifies such study.

\section{Acknowledgment}

David S. Johnson, the lead author of this paper, passed away on March 8, 2016. David's co-authors dedicate this paper to his memory. The authors thank David Applegate for helpful discussions and in particular for simplifying the statement and proof of Theorem 5 . The authors also thank Rodrigo Toso for implementing the version of Genetic used in the experiments.

\section{References}

A. V. Aho, J. E. Hopcroft, and J. D. Ullman. The design and analysis of computer algorithms. Addison-Wesley Publishing Company, Reading, Massachusetts, 1974.

N. Alon, D. Moshkovitz, and S. Safra. Algorithmic construction of sets for $k$-restrictions. $A C M$ Transactions on Algorithms, 2:153-177, 2006.

J. Bean. Genetics and random keys for sequencing and optimization. ORSA Journal on Computing, 6:154-160, 1994.

M. Bellare, S. Goldwasser, C. Lund, and A. Russell. Efficient probabilistic checkable proofs and applications to approximation. In Proceedings of the 25th Annual ACM Symposium on the Theory of Computing, pages 294-304, New York, NY, 1993. ACM.

L. Breslau, C. Chase, N. Duffield, B. Fenner, Y. Mao, and S. Sen. Vmscope - A virtual multicast VPN performance monitor. In ACM SIGCOMM Workshop on Internet Network Management (INM), pages 59-64, 2006. 
L. Breslau, I. Diakonikolas, N. Duffield, Y. Gu, M. Hajiaghayi, D. S. Johnson, H. Karloff, M. G. C. Resende, and S. Sen. Disjoint-path facility location: Theory and practice. In Proceedings of the 11th Annual Workshop on Algorithm Engineering and Experimentation (ALENEX), pages 60-74, 2011.

H. Burch and C. Chase. Monitoring link delays with one measurement host. SIGMETRICS Performance Evaluation Review, 33(3):10-17, 2005.

L. S. Buriol, M. G. C. Resende, and M. Thorup. Survivable IP network design with OSPF routing. Networks, 49(1):51-64, 2007.

K. Calvert, M. Doar, and E. Zegura. Modeling internet topology. IEEE Communications Magazine, 35(6):160-163, 1997.

U. Feige. A threshold of $\ln n$ for approximating set cover. Journal of the ACM, 45:634-652, 1998. Preliminary version in Proceedings of the 28th Annual ACM Symposium on the Theory of Computing, ACM, New York, 1996, 314-318.

B. Fortz and M. Thorup. Internet traffic engineering by optimizing OSPF weights. In Proceedings of INFOCOM 2000: 19th Annual Joint Conference of the IEEE Computer and Communications Societies, pages 519-528. IEEE, 2000.

J. F. Gonçalves and M. G. C. Resende. Biased random-key genetic algorithms for combinatorial optimization. Journal of Heuristics, 17:487-525, 2011.

Y. Gu, L. Breslau, N. Duffield, and S. Sen. GRE encapsulated multicast probing: A scalable technique for measuring one-way loss. In IEEE Infocom 2008: The 27th Conference on Computer Communications, pages 1651-1659, New York, NY, 2008. IEEE Communications Society.

R. Hassin and D. Segev. The set cover with pairs problem. In FSTTCS 2005: Proceedings of the 25th International Conference on Foundations of Software Technology and Theoretical Computer Science, volume 3821 of Lecture Notes in Computer Science, pages 164-176, Berlin, 2005. Springer-Verlag.

D. S. Johnson. Approximation algorithms for combinatorial problems. Journal of Computer and System Sciences, 9:256-278, 1974.

G. Kortsarz. On the hardness of approximating spanners. Algorithmica, 30:432-450, 2001.

L. Lovász. On the ratio of optimal integral and fractional covers. Discrete Mathematics, 13:383-390, 1975.

R. Raz and S. Safra. A sub-constant error-probability low-degree test, and a sub-constant errorprobability PCP characterization of NP. In Proceedings of the 29th Annual ACM Symposium on the Theory of Computing, pages 475-484, New York, NY, 1997. ACM. 